\title{
Horizontal Ampoule Growth and Characterization of Mercuric Iodide at Controlled Gas Pressures for X-Ray and Gamma Ray Spectrometers
}

\author{
(DOE NEER Grant Number 00-ID-13919)
}

Douglas S. McGregor, Elsa Ariesanti, Bridget Corcoran

Semiconductor Materials and Radiological Technologies Laboratory

Department of Nuclear and Mechanical Engineering

Kansas State University

Manhattan, KS 66506
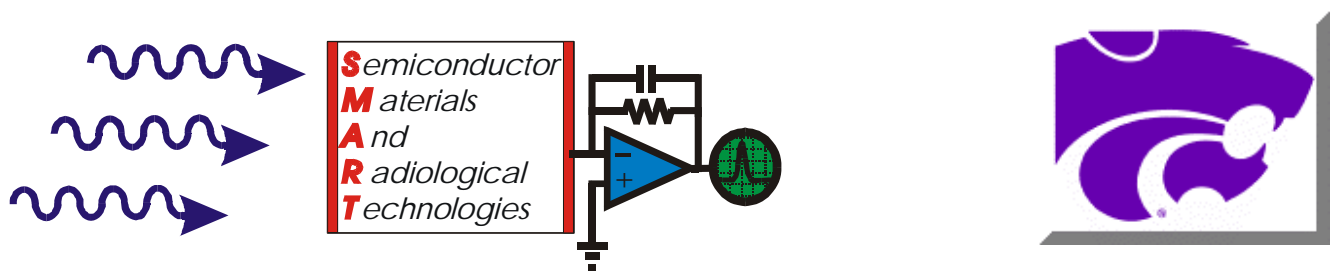

Submitted to the

Department of Energy

Nuclear Engineering and Educational Research Program

April 2004 
Progress Report for year 3 covering the period

June, 2003 - May, 2004

\title{
Horizontal Ampoule Growth and Characterization of Mercuric Iodide at Controlled Gas Pressures for X-Ray and Gamma Ray Spectrometers
}

\author{
Douglas S. McGregor, Elsa Ariesanti, Bridget Corcoran \\ SMART Laboratory, Kansas State University, Manhattan, KS 66506
}

\section{Introduction}

The following described DOE sponsored Nuclear Engineering Educational Research (NEER) program addresses a new method for producing high quality mercuric iodide $\left(\mathrm{HgI}_{2}\right)$ crystals for radiation spectrometers. $\mathrm{HgI}_{2}$ is a semiconductor material with a relatively wide band gap of $2.13 \mathrm{eV}$. The wide band gap serves to significantly reduce thermal leakage current noise at room temperature. Additionally, the elements comprising the material have high atomic numbers, being 53 for iodine and 80 for mercury, important physical attributes that significantly increase the gamma-ray absorption probability over other semiconductor materials that have been traditionally used, such as $\mathrm{Ge}$ and $\mathrm{Si}$. For instance, a piece of $\mathrm{HgI}_{2}$ only $2 \mathrm{~mm}$ thick will absorb the same fraction of $100 \mathrm{keV}$ gamma rays as will a $2 \mathrm{~cm}$ thick piece of Ge [1].

Yet, $\mathrm{HgI}_{2}$ comes with its own unique set of difficulties. Unlike common semiconductors used in the VLSI industry, $\mathrm{HgI}_{2}$ is non-cubic, forming in a tetragonal crystal structure (noted as the $\alpha$-phase) at temperatures below $130^{\circ} \mathrm{C}$. At temperatures above $130^{\circ} \mathrm{C}$, the crystal undergoes a phase change and forms in an orthorhombic crystal structure, noted as the $\beta$-phase, and if cooled below $130^{\circ} \mathrm{C}$ destructively turns to the $\alpha$-phase $[1,2]$. Hence, the crystal must be grown such that the material does not crystallize at temperatures above $130^{\circ} \mathrm{C}$ along with the additional constraint that detectors fabricated from the material must not be stored or operated in environments that may allow for the critical temperature of $130^{\circ} \mathrm{C}$ to be reached or exceeded.

As with any semiconductor used for gamma radiation spectroscopy, reduction of impurities is of major importance. The presence of impurities causes the appearance of trapping sites, hinder the transport properties of charge carriers, and cause distortions in the electric field distribution. Thus the production of high-quality $\mathrm{HgI}_{2}$ spectrometers requires 
careful handling of the material during pre-growth, growth, and post-growth stages, since impurities may be introduced in some or all of these stages.

A method by S. Faile in 1981 has been widely used to produce $\mathrm{HgI}_{2}$ platelets in horizontal furnaces $[3,4]$. The Faile method promises to produce good quality platelets in a short growth period (within days) with the use of organic materials to enhance the properties of the platelets. To date, however, the role of the organic materials during the actual growth process has not been understood. In the mean time, efforts to obtain high-quality crystals without the use of organic materials have also been reported with encouraging results [5]. Goals of the present project include (1) development of a new method to produce multiple $\mathrm{HgI}_{2}$ crystals for small radiation spectrometers, (2) characterization of $\mathrm{HgI}_{2}$ crystal properties as a function of growth environment, and (3) fabrication and demonstration of roomtemperature-operated high-resolution $\mathrm{HgI}_{2}$ spectrometers.

\section{Experimental Arrangements and Installations}

The initial phase of the project partially consisted of the specification, ordering, and delivery of equipment and materials required for the success of the project. Included are the acquisition of a de-ionizing water system, a vacuum evacuation ampoule system, customized growth furnaces, stock $\mathrm{HgI}_{2}$ starting material, and various smaller parts and growth accessories. The project was originally started at the University of Michigan (UM). Unfortunately, UM bidding and construction rules caused numerous delays with program progress; hence the acquisition of many materials was realized late in the first year of the program. An additional six month delay was also caused by the following factors; 1) bidding and purchasing agreement delays for the DI water system, 2) delays with the construction and plant department regarding safety concerns over furnace and pumping installations, and 3) delivery delays for the various equipment after the orders were placed. Overall, over a year of lost time resulted from the litany of problems. Ultimately, the PI (McGregor) chose to relocate the research to another institution (Kansas State University) to reduce the probability any further problems. The relocation of the Semiconductor Materials and Radiological Technologies (SMART) Laboratory to Kansas State University (KSU) added further delay to the project. Although the cooperative atmosphere of KSU made the transition workable, it 
was practically a year before the SMART Laboratory was in operation again. Regardless of the delays, meaningful advances have been achieved, as will be described.

The results obtained in Phase I of the project confirmed the necessity of obtaining starting material with high purity to ensure growth of high quality $\mathrm{HgI}_{2}$ single crystals. The proposed research plan for the remainder of the project as described in Phase I progress report has been modified by including the following stages in the current research plan: (1) synthesis of starting material from aqueous solutions of potassium iodide (KI) and mercuric chloride $\left(\mathrm{HgCl}_{2}\right)$, and (2) vapor growth of single crystals in vertical furnaces (or commonly known as the "EG\&G method"). In addition, new equipment has been acquired to upgrade previously employed equipment and systems. Descriptions of the acquisitions, arrangements, and installations follow.

\section{A. Acquisitions}

A reverse osmosis deionizing (DI) water system was ordered, relocated, and installed at the SMART Laboratory at KSU. The DI system has a 300-gallon storage tank in which the water is constantly circulated through the system loop and filtered, thereby preventing stagnation and microorganism accumulation. The system also has an ultraviolet filter to dispatch any living organisms that may enter the water loop. A series of filters, the smallest being 0.2 microns, cleans the water to produce $18 \mathrm{M} \Omega-\mathrm{cm}$ resistivity water.

Over forty horizontal furnaces have been designated solely to the project. Most of the furnaces were donated to the SMART Laboratory by Renaissance Instruments of Round Rock, TX, renowned for their work in horizontal growth of $\mathrm{HgI}_{2}$ crystals. The furnaces acquired include forty two-zone horizontal furnaces, two transparent Pyrex two-zone purification furnaces, and six vertical furnaces. Further, Sandia National Laboratories donated one two-zone sublimation purification furnace, four vertical furnaces, a turbo pumping system, and several pounds of purified stock $\mathrm{HgI}_{2}$ material. All such furnaces have been installed in the KSU SMART Laboratory.

The numerous furnaces acquired require two thermocouple temperature controllers

each. Due to budget restrictions, only a portion of the furnaces could be installed and brought into operation. At present, four vertical furnaces are operating, ten horizontal furnaces are operating, and one sublimation purification furnace is operating. Other 
donations to the project came from MOXTEK, of Orem, Utah, who donated several pounds of raw $\mathrm{HgI}_{2}$ stock material. MOXTEK also donated numerous temperature controllers and furnace tubes.

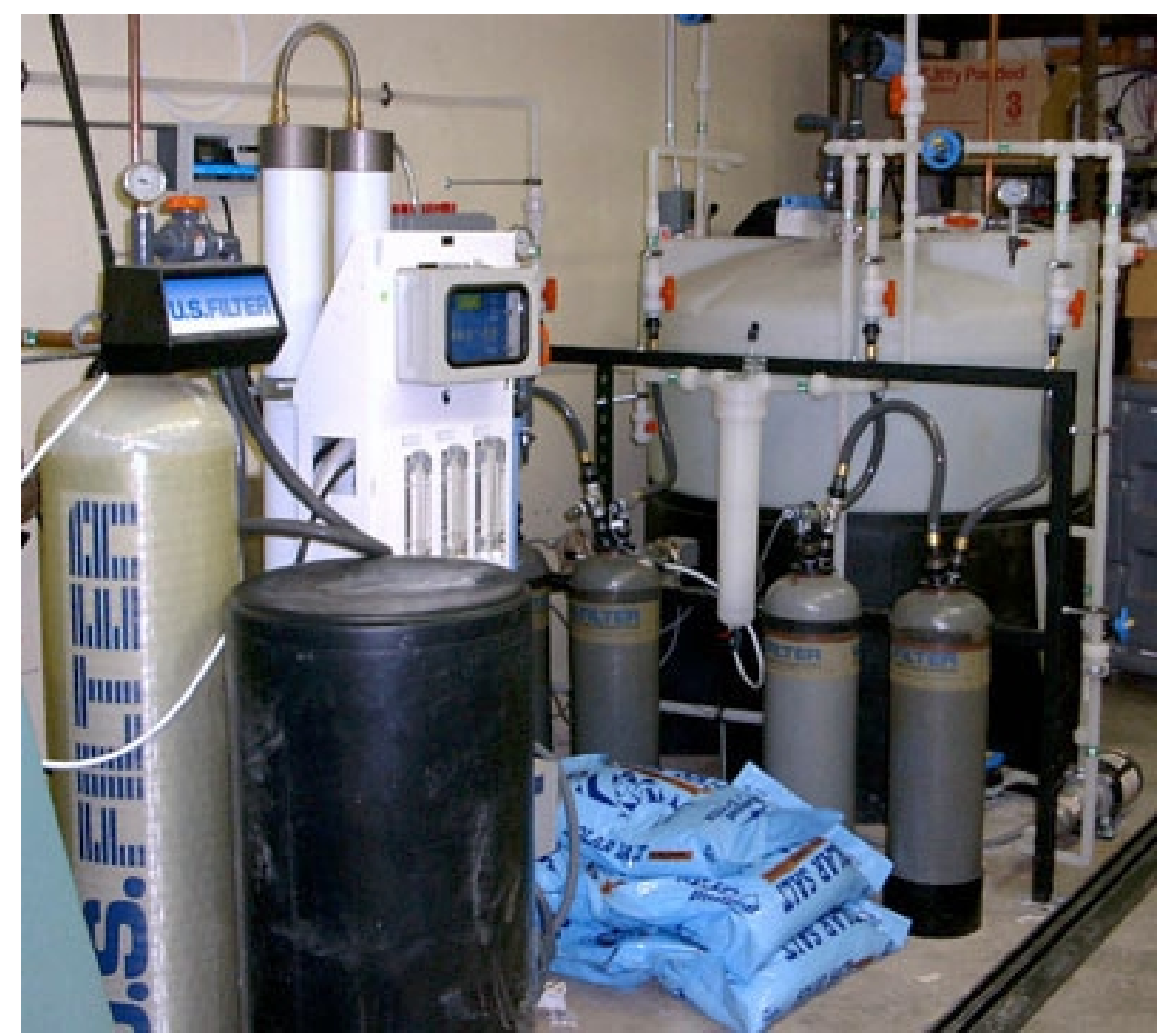

Figure 1: Reverse osmosis de-ionizing system installed in the SMART Laboratory. The system produces 18.6 mega-ohm $(\mathrm{M} \Omega$ ) resistivity water and has a 300 -gallon storage tank.

Early generation ampoules for $\mathrm{HgI}_{2}$ material growth were two inches in diameter with two glass suspension rods at each end, one of which was hollow and open. The ampoules also had an open tubular glass side port that has been necked down. The end port opening was for loading $\mathrm{HgI}_{2}$ material into the ampoule and the side port was for vacuum evacuating the ampoule. Early generation ampoules for $\mathrm{HgI}_{2}$ material purification, one-inch diameter and five-foot long, were composed of Pyrex tubes with quick connect vacuum outlet on one end. The long ampoules were stationed in long four-inch diameter furnaces (except during cleaning procedures). Open-ended cylindrical glass boats were used for the purification process (described later). 


\section{B. Installations}

\section{Ampoule pumping station}

A gas handling manifold and purification system has been constructed to evacuate and inject selected gases into the growth ampoules. The ampoule station consists of a gashandling manifold that employs hydrogen, argon, oxygen, nitrogen, and hydrochloric acid gases and a pumping system, which can evacuate ampoules to at least $1 \times 10^{-5}$ Torr. Thus evacuation and gas back filling of ampoules can be accomplished in one setting. The system is hooked to a vacuum pump, thus allowing for the $\mathrm{HgI}_{2}$ growth ampoule to be vacuum evacuated and subsequently filled with a process gas before final closure. A vacuum meter is integrated with the system to accurately determine the vacuum and gas pressure.

Each gas used in the manifold is fed through a gas-specific purifier before reaching an ampoule. A dual-stage roughing pump is used to initially evacuate the ampoule. Then subsequently the ampoule is evacuated using two sorption pumps (one is employed after the other). When the desired vacuum level is achieved, the ampoule is sealed. A dual-port vacuum ampoule station capable of sealing much larger diameter ampoules has been attached to the original station. The vacuum ampoule station now has three separate ports to accommodate various ampoule diameters. High purity Teflon lines connect the backfill gas lines to all pumping ports.

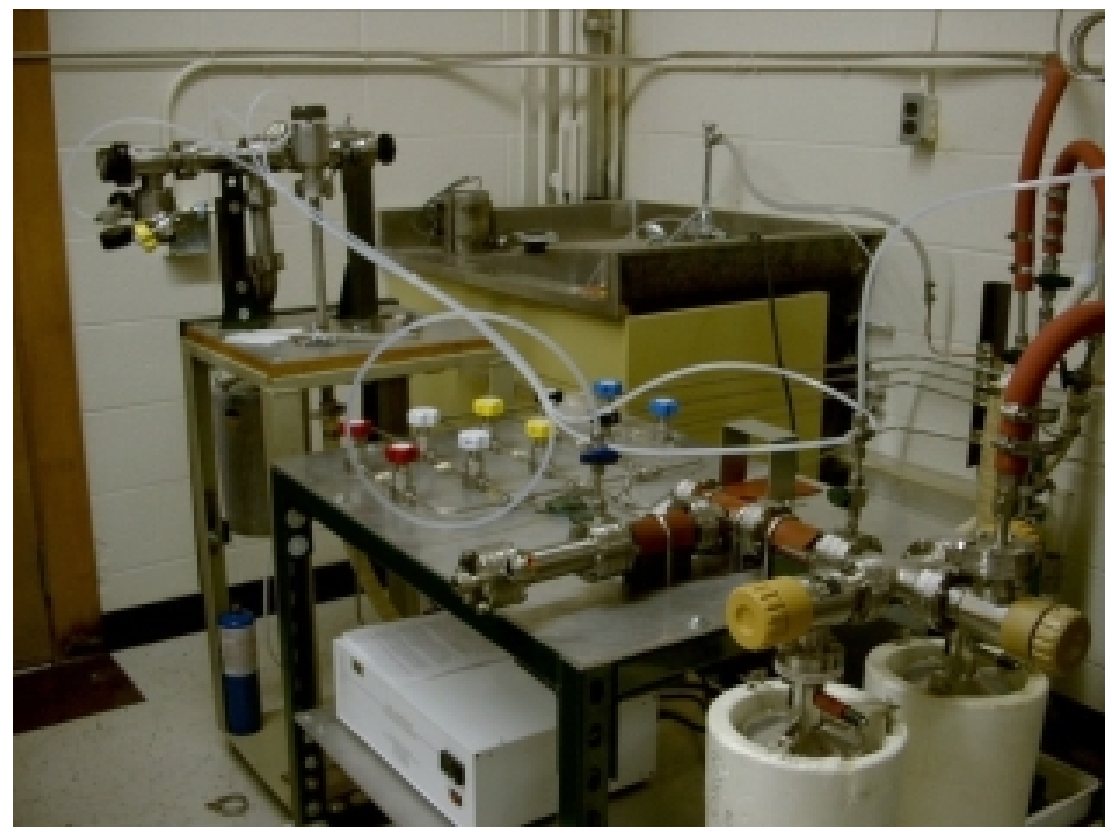

Figure 2: The multi-station ampoule station designed and built to seal the $\mathrm{HgI}_{2}$ filled ampoules for purification and crystal growth. 


\section{Sublimation Purification Furnace}

A single-zone sublimation purification furnace donated by Sandia National Laboratories has been installed and is operating. The zone is controlled with a temperature controller and K-type thermocouple. The purification ampoule utilized is 28 inches long and 3.5 inches in diameter, which is significantly larger in volume than previous purification ampoules uses in the early stages of the project. The ampoule is sealed with an O-ring and cap (see Figure 3). A Teflon sleeve is located at one end of the ampoule near the O-ring seal. The sealed portion of the ampoule where the Teflon sleeve is located extends outside the insulated furnace, hence the temperature is significantly lower, thereby causing purified $\mathrm{HgI}_{2}$ vapor to redeposit inside the Teflon sleeve. A cold trap it located beyond the cool region (where the Teflon sleeve is) and before the pumping stages. The pumping stages consist of a rotary vane roughing pump and a high-vacuum turbo pump.

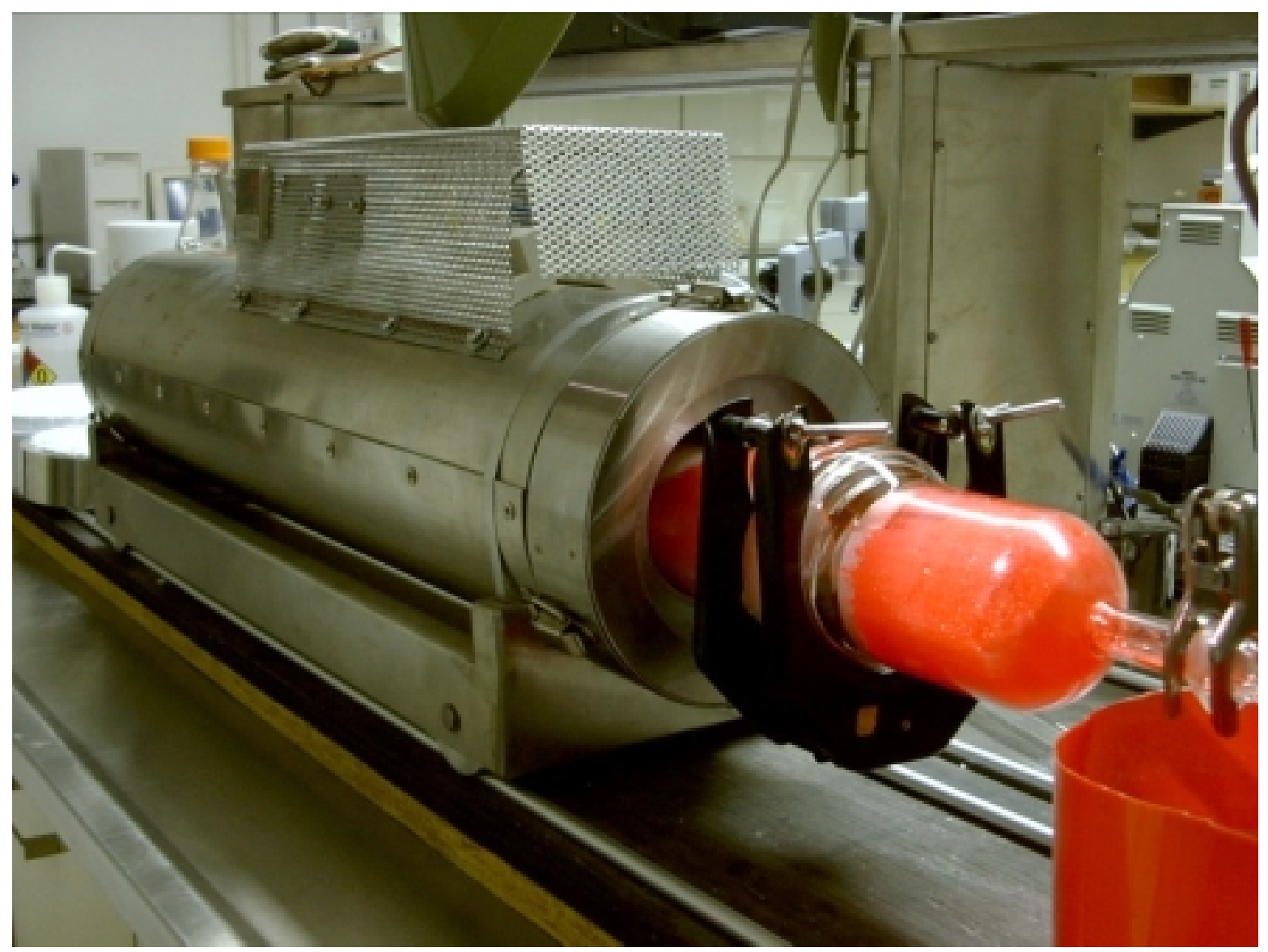

Figure 3: The two-zone $\mathrm{HgI}_{2}$ sublimation purification furnace used to separate impurities from stock $\mathrm{HgI}_{2}$. Good $\mathrm{HgI}_{2}$ material is collected from the end protruding from the furnace. 
The device operates by subliming stock $\mathrm{HgI}_{2}$ material and transporting $\mathrm{HgI}_{2}$ molecules to the cooler region, where they are redeposited. Impurities are left in the high temperature region, and volatiles are transported to the cold trap. The purified $\mathrm{HgI}_{2}$ material is collected from the Teflon collar in the cool region. Generally it takes at least at least three sublimation runs to complete this stage in the material purification process.

\section{Zone Refiner}

A furnace designated for zone refinement processing has been designed and built. The zone refinement process has been used successfully to remove impurities that are generally not removable by other purification methods. One of the purposes of this process is to purify $\mathrm{HgCl}_{2}$ and $\mathrm{KI}$, which are the starting material for the $\mathrm{HgI}_{2}$ synthesis. Aqueous solutions $\mathrm{HgCl}_{2}$ and $\mathrm{KI}$, each made by mixing highly purified de-ionized water and the chemicals, are brought together in a large reaction vessel. The resulting reaction produces solid $\mathrm{HgI}_{2}$ particles. Hence, it is imperative that the starting materials are purified first.

The zone refiner is shown in Figure 4. The zone refiner has three quartz tubes for insulation and heat confinement (see Figure 5). A sample to be purified is sealed in an ampoule. The ampoule is placed inside the innermost tube and connected to a stepping motor with high temperature resistant string. The innermost quartz tube has single thin Nichrome wire loop wrapped around region in the middle. The thin heated region is used to melt the stock material, and is maintained at a temperature slightly above the melting point of the material. A second quartz tube that is placed over the inner tube confines the temperature. To maintain a controlled heating environment, the second (or middle) tube has a thicker Nichrome heating coil wrapped around it from end to end. The middle tube is heated such that it is slightly below the melting point of the material to be purified.

Lastly, to reduce heat loss, a third quartz tube is placed over the middle tube. Insulating blocks at each end serve to reduce heat loss from the ends and to maintain a rigid structure. The furnace tube arrangement is placed on an aluminum plate that can be slanted at a pre-determined angle to enhance material transfer. A small, evacuated, and sealed quartz/Pyrex tube (filled with material to be purified) is then pulled uphill along the length of the furnace by a motor at a pre-determined speed. 


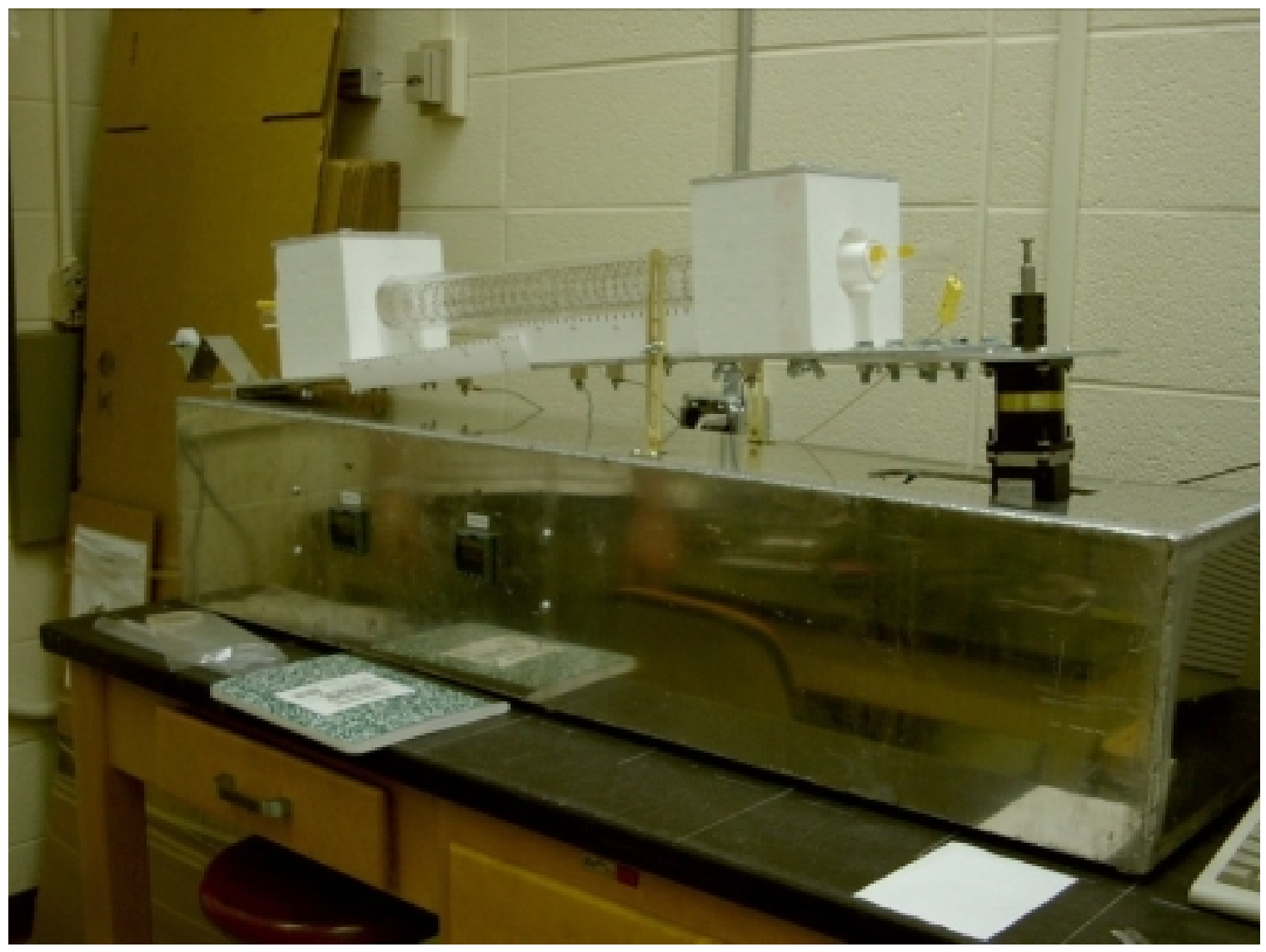

Figure 4: Zone refiner that was built to purify $\mathrm{HgCl}_{2}$ and $\mathrm{KI}$ starting materials, and $\mathrm{HgI}_{2}$ material.

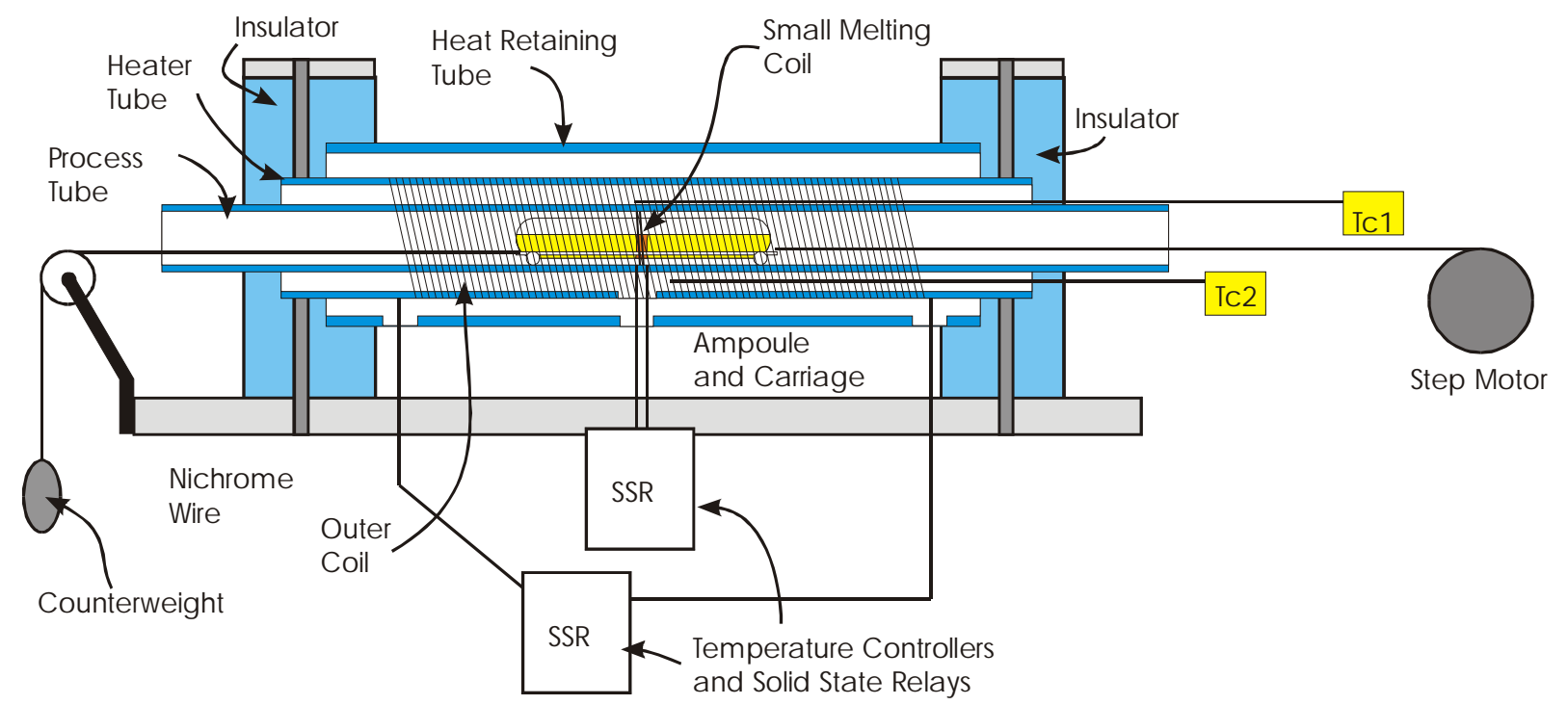

Figure 5: The zone refiner configuration, showing the separate zones. 
The purification process works by slowly pulling the sample to be processed through the thin melting zone. Impurities migrate away from the melted region, which slowly pushes the impurities to the ends of the entire material sample. Numerous passes must be performed for a sample, ranging from ten to over thirty. The zone refiner pull rate is controlled with LabView and a computer. The zone refiner is used (a) to purify starting materials for the initial $\mathrm{HgI}_{2}$ synthesis, and (b) as a last purification step of the $\mathrm{HgI}_{2}$ material before placing the product in the growth ampoules.

\section{Horizontal Furnaces}

The crystal growth method used for most of the project is based on the horizontal technique first introduced by Faile [3,4]. As mentioned earlier, Renaissance Instruments donated forty horizontal furnaces to the project [6]. The furnaces all need two temperature controllers each capable of $0.1^{\circ} \mathrm{C}$ control resolution. Only ten of the horizontal furnaces have been outfitted with temperature controllers and are available for crystal growth. Each furnace has two heating zones with a total combined length of 20 inches.

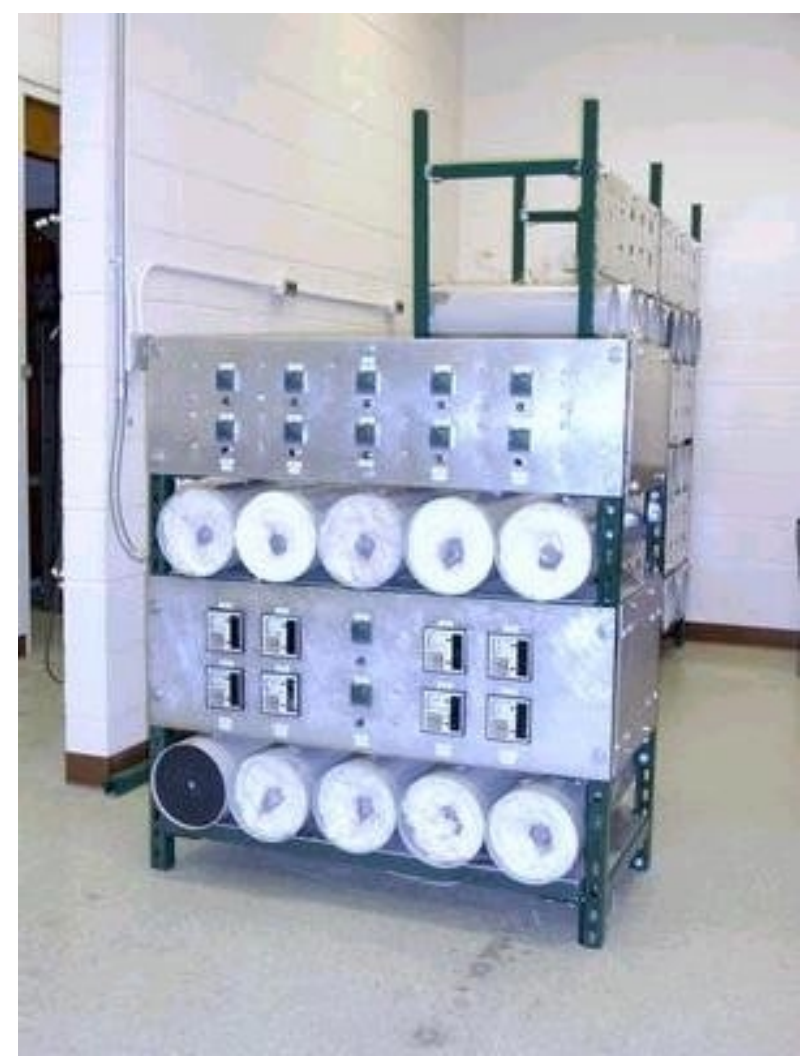

Figure 6: A bank of ten horizontal two-zone furnaces used for $\mathrm{HgI}_{2}$ crystal growth. In the background can be seen another thirty horizontal furnaces. 


\section{Vertical Furnaces}

Another crystal growth method under investigation for the project is based on the vertical technique first introduced by Sholtz [7]. Sandia National Laboratories donated four vertical furnaces to the project, and Renaissance Instruments donated another six. The furnaces all need two temperature controllers each capable of $0.1^{\circ} \mathrm{C}$ control resolution. Only four of the furnaces have been outfitted with temperature controllers and are available for crystal growth. Each furnace has two controllers to vary the temperature profile.

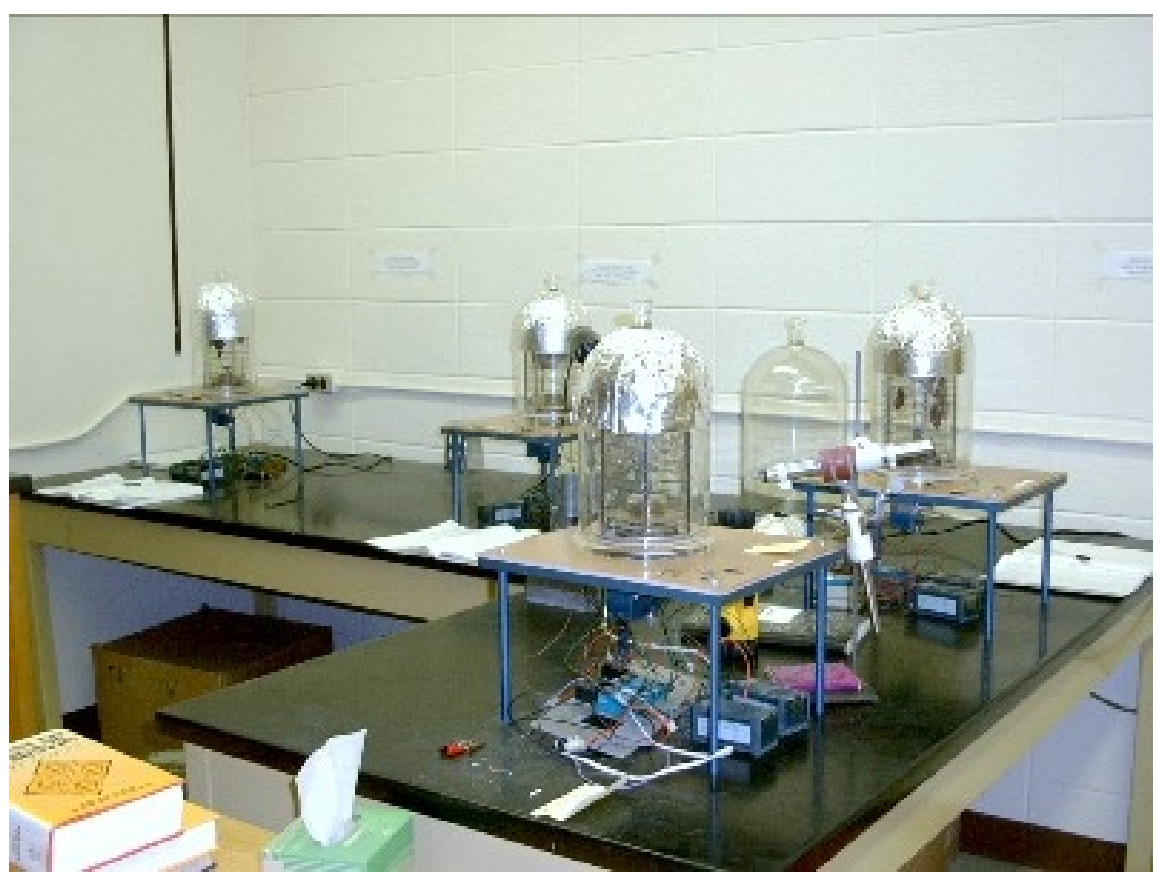

Figure 7: Vertical furnaces used for $\mathrm{HgI}_{2}$ crystal growth.

\section{Synthesis Hood}

An eight-foot long chemical hood was purchased and installed to perform the $\operatorname{HgI}_{2}$ synthesis in. The long quartz and Pyrex tubes used in the eight-foot furnaces are cleaned in a high-density polypropylene (HDPE) dual troth that can be stationed in the eight-foot hood when needed. The glassware is cleaned and etched in various acids before material is sealed in them. The cleaning steps are necessary to avoid contamination of unwanted impurities in the material before and during crystal growth. 


\section{Experimental Methods and Results}

\section{A. Review of the Previous Phase}

All growth and processing were performed at the Semiconductor Materials and Radiological Technologies (S.M.A.R.T.) Laboratory. Stock $\mathrm{HgI}_{2}$ was loaded by inserting a long-stemmed funnel into a growth ampoule end tube opening and pouring a pre-measured amount of stock material into the ampoule. The long stem ensures that the $\mathrm{HgI}_{2}$ is deposited at the bottom of the ampoule. The ampoule end tube is then sealed at the tip and the side evacuation tube is connected to the vacuum evacuation system. After reaching the predetermined vacuum for the growth experiment (and gas environment for future growths), the ampoule is sealed. The ampoule is then suspended within the small three-zone furnace by the glass suspension rods (see Figure 8). All growth runs were conducted in a vacuum. The early runs were conducted to determine the best parameters for growing reasonably large crystals.

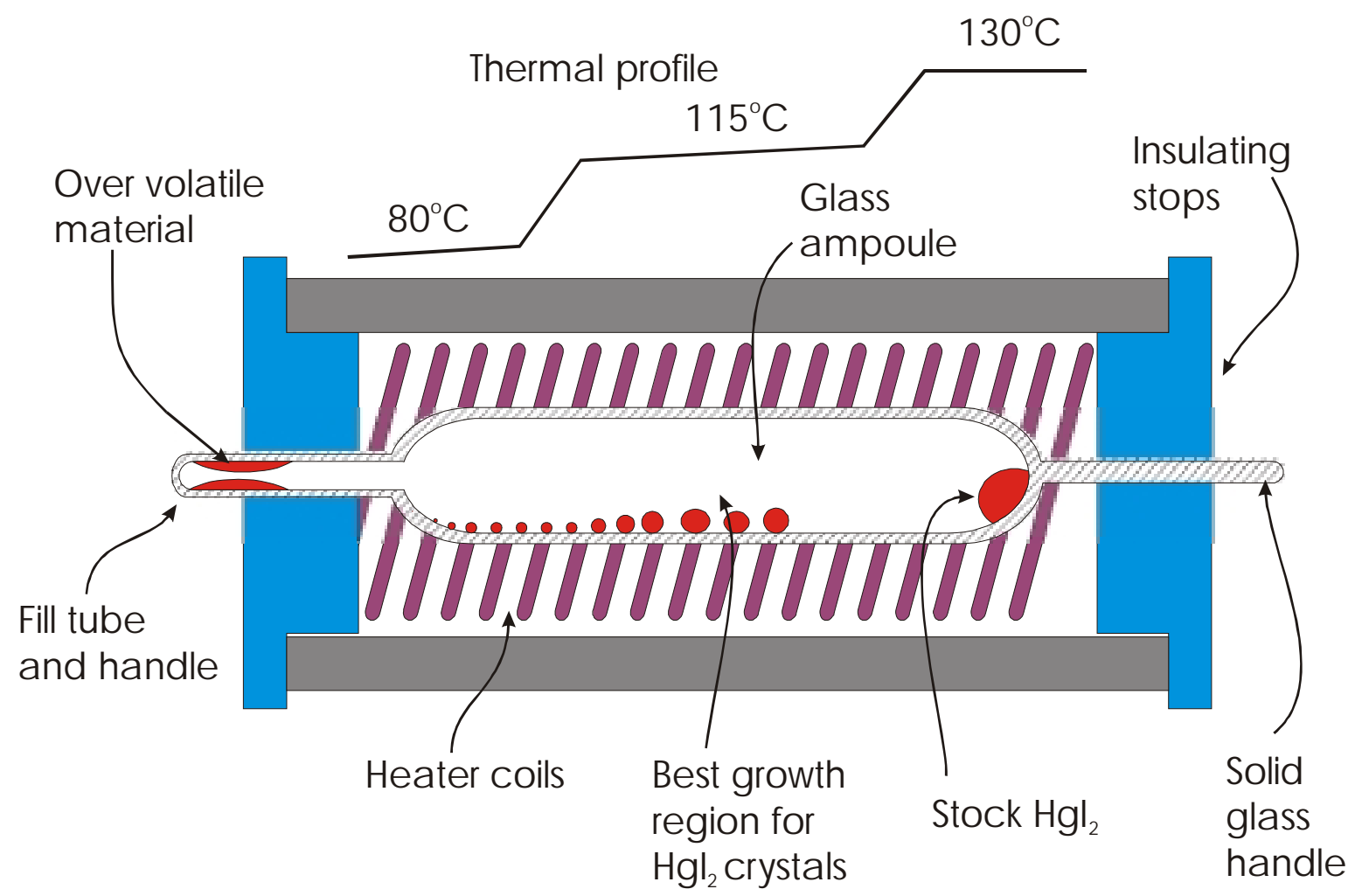

Figure 8: The growth furnace arrangement used in phase I.

Early experiments have shown that the growth starting conditions are important to the successful growth of relatively large crystals. Early growth runs were conducted by inserting 
the ampoules into a furnace that was already warmed to the desired thermal profile. The early thermal profiles had the following settings: End Zone 1 (six inches long) $=200^{\circ} \mathrm{C}$, Middle Zone 2 (12 inches long) $=130^{\circ} \mathrm{C}$, End Zone 3 (six inches long) $=90^{\circ} \mathrm{C}$. The ampoule was allowed to rest in the furnace for 5 days, after which is was removed for examination. Figure 9 shows the results from an early growth run. It should be noticed that the $\mathrm{HgI}_{2}$ crystals are virtually microscopic, which is interesting for study, but impractical for radiation detector development. It became clear after the results were repeated several times that the growth parameters had to be changed.

The first change made was the thermal profile, which was lowered for many growths with a variety of profiles. For instance, many growths had the following profile: End Zone 1 $=170^{\circ} \mathrm{C}$, Middle Zone $2=115^{\circ} \mathrm{C}$, End Zone $3=90^{\circ} \mathrm{C}$. Simply lowering the temperature continued to produce results very similar to the crystals shown in Figure 9. Hence, lowering the temperature of the growth did not adequately address the problem.

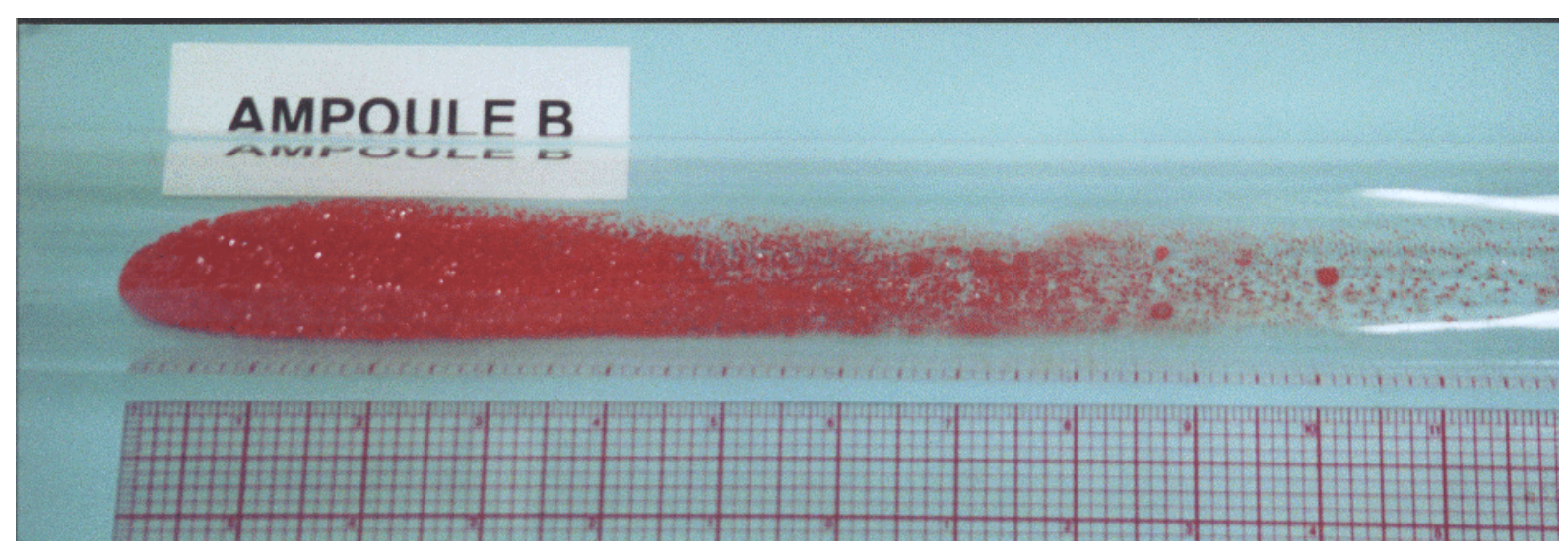

Figure 9: Mercuric iodide crystals grown in a preheated furnace, in which the furnace was heated to the growth temperatures before the ampoule was inserted (End Zone $1=200^{\circ} \mathrm{C}$, Middle Zone $2=130^{\circ} \mathrm{C}$, End Zone $3=90^{\circ} \mathrm{C}$ ). The crystals shown are small in size and impractical for gamma-ray detector use.

The initial starting parameters were then changed. The ampoules were loaded into a cold furnace, and the thermal profile was slowly brought up to the growth temperatures of: End Zone $1=160^{\circ} \mathrm{C}$, Middle Zone $2=105^{\circ} \mathrm{C}$, End Zone $3=90^{\circ} \mathrm{C}$. The slowly warmed ampoule allowed for crystals to nucleate and grow much larger, as shown in Figure 10.

The process was further improved by changing the initial starting material from powder form to solid form, thereby reducing the material sublimation rate and the resulting 
crystal nucleation and growth rate. Solidification consisted of a two-step process. First, a sealed ampoule was placed in the furnace and the starting $\mathrm{HgI}_{2}$ powder material was sublimed and vapor transported from the end to the ampoule center where it condensed. Afterwards, the thermal profile was changed such that the $\mathrm{HgI}_{2}$ material was driven back to the end, whereby it condensed as a solid piece. The ampoule was then removed from the furnace and the furnace was allowed to cool down to room temperature. The growth procedure, as previously described, was then implemented, with a reduced lower thermal profile (End Zone $1=135^{\circ} \mathrm{C}$, Middle Zone $2=115^{\circ} \mathrm{C}$, End Zone $3=90^{\circ} \mathrm{C}$ ). The resulting crystals, shown in Figure 11, were larger than previous runs. However, it was noticed that many of the large crystals formed in the $\beta$-phase, as was indicated by their yellow color when the ampoule was removed from the furnace. The large crystals reformed in the $\alpha$-phase as they cooled, causing the crystals to form as polycrystalline.

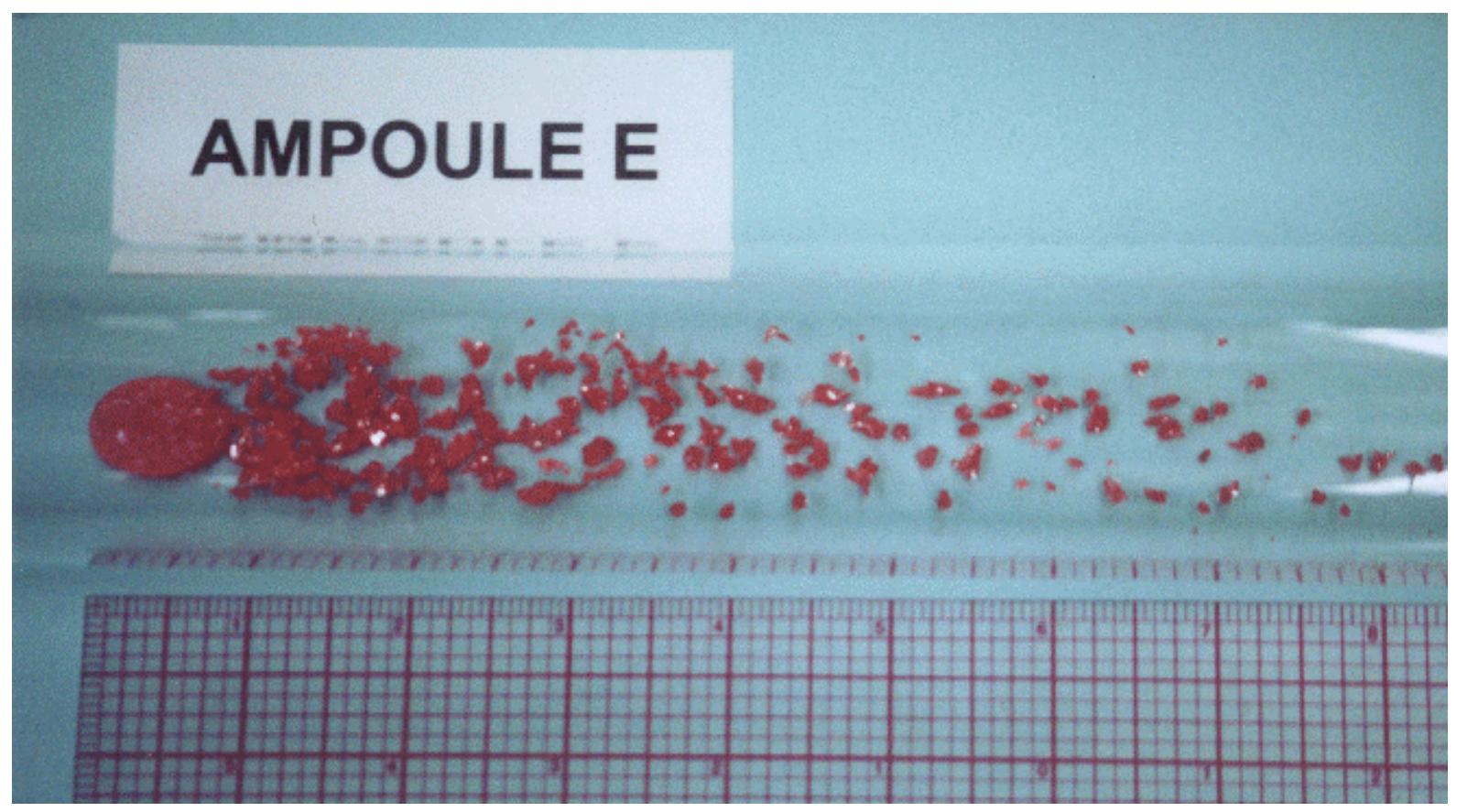

Figure 10: Mercuric iodide crystals grown with slow thermal warming and lowered furnace temperatures. The furnace was heated to the growth temperatures after the ampoule was inserted (End Zone $1=160^{\circ} \mathrm{C}$, Middle Zone $2=105^{\circ} \mathrm{C}$, End Zone $3=90^{\circ} \mathrm{C}$ ). The crystals shown are larger than the crystals grown with preheated furnace conditions.

The technique was repeated for another sample, where the stock $\mathrm{HgI}_{2}$ powder was resublimed into a solid mass at the end of the ampoule. The slow warming growth procedure was then implemented, with the thermal profile further reduced to prevent the formation of 
$\beta$-phase crystals in the growth zone (End Zone $1=120^{\circ} \mathrm{C}$, Middle Zone $2=100^{\circ} \mathrm{C}$, End Zone $3=80^{\circ} \mathrm{C}$ ). The resulting crystals, shown in Figure 12, were the largest grown thus far, with diameters being larger than $6 \mathrm{~mm}$ for many. Crystals of this size (5 $\mathrm{mm}$ or more) are large enough to evaluate material properties and make useful X-ray and low energy gamma-ray detectors. Crystal twinning is apparent on some samples, but polycrystalline samples did not form.

\section{AMPOULE G}

-

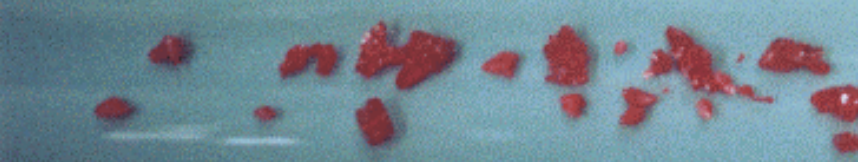

$\sin 2$

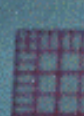

Figure 11: Mercuric iodide crystals grown with slow thermal warming at even lower temperatures (End Zone $1=135^{\circ} \mathrm{C}$, Middle Zone $2=115^{\circ} \mathrm{C}$, End Zone $3=90^{\circ} \mathrm{C}$ ). The crystals showed markedly larger sizes than previous runs, indicating that lower temperatures are better. The crystals show obvious signs of twinning and polycrystalline regions most likely due to $\beta$-phase formation in the growth zone.

It was also noticed that a yellow and orange residue remains at the source end of the ampoule. Although the starting material was labeled as high purity (acquired from AlfaAesar), the residue is a deposit of impurity material left behind by the vapor transport growth. Hence, the starting material is contaminated with excessive impurities. It is necessary to implement a purification process for the $\mathrm{HgI}_{2}$ material before the growth ampoules are loaded and sealed. Condensed matter was found inside of the hollow glass fill handle as well, which is assumed to be the more volatile portions of the stock $\mathrm{HgI}_{2}$ material. Purification will reduce the quantity of the over-volatile material. 


\section{AMPOULE H}
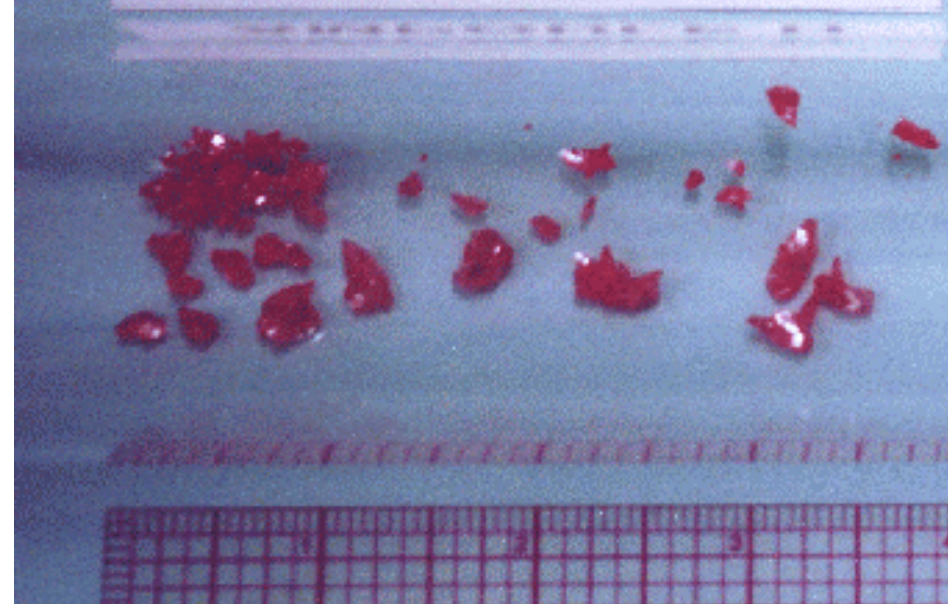

Figure 12: Mercuric iodide crystals grown from a solid source and with slow thermal warming. The temperatures were reduced to prevent the formation of $\beta$-phase crystals in the growth zone (End Zone $1=120^{\circ} \mathrm{C}$, Middle Zone $2=100^{\circ} \mathrm{C}$, End Zone $3=80^{\circ} \mathrm{C}$ ). The crystals again showed markedly larger sizes than previous runs, and the crystallinity appears much better, showing much fewer twinned regions. Such crystals have large enough regions that can be used for small gamma ray spectrometers.

\section{B. Present Phase}

Overall, it was observed that a slowly warming ampoule produced the largest crystals. A new growth design was implemented in the next growth run, in which glass capillary tubes were inserted into the furnace beneath the growth ampoule. The glass tubes had nozzles pointing towards the ampoule, and dry nitrogen will be forced through the capillaries such that they cooled pre-appointed spots (or regions) on the ampoule wall. The cooling provided a zone were the $\mathrm{HgI}_{2}$ would selectively grow while further slowing down the temperature increase. It was observed that largest crystals grew in a region of approximately $115^{\circ} \mathrm{C}$, hence the nitrogen-cooled spots were located to meet that criteria.

The starting $\mathrm{HgI}_{2}$ material was purified in the following fashion. Purification begins with stock $\mathrm{HgI}_{2}$ powder material being loaded into a small glass boat and inserted into a 5foot long narrow glass cylinder. The material was heated such that it sublimes and then condenses at the far end of the cylinder into a receiver boat, thereby leaving behind deleterious impurities (see Figure 13). The receiver boat was inserted into another five-foot purification tube and the process is repeated. Sublimation purification was performed several 
times and the resulting material is harvested. The harvested material was then sealed into a small glass ampoule. A thorough baking procedure is implemented at a high enough temperature $\left(300^{\circ} \mathrm{C}\right)$ to melt the $\mathrm{HgI}_{2}$ such that residual impurities are further removed from the material. The resulting material is then removed from the small ampoule. A last step includes multiple zone refinement of the final product. After these steps, the material is ready for crystal growth.

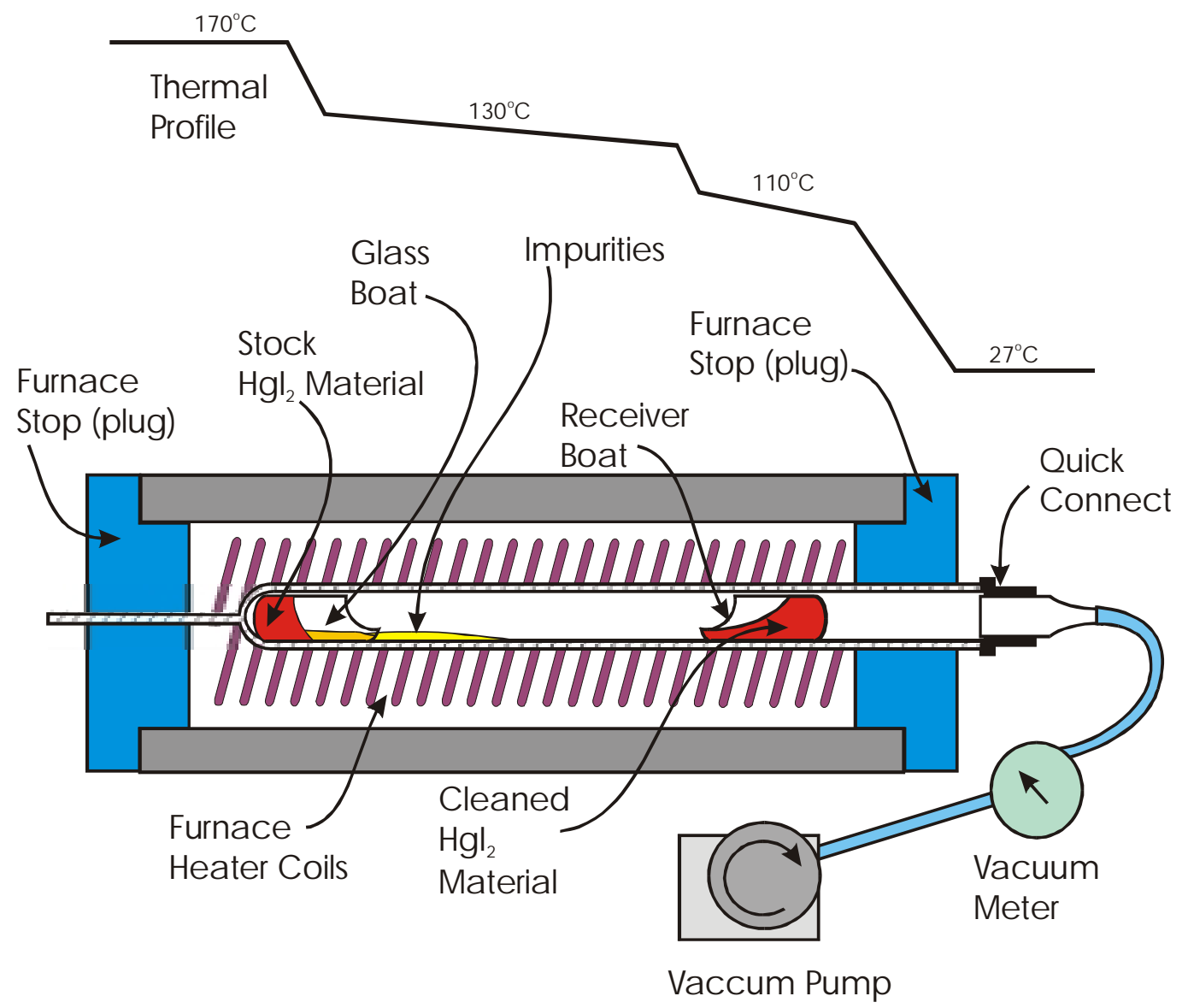

Figure 13: The initial purification furnace arrangement.

\section{Synthesis of material}

Synthesis of mercuric iodide from aqueous solutions of potassium iodide (KI) and mercuric chloride $\left(\mathrm{HgCl}_{2}\right)$ are being pursued. This process has been described in literature and the results are preferable compared to commercially synthesized $\mathrm{HgI}_{2}$. Commercially synthesized $\mathrm{KI}$ and $\mathrm{HgCl}_{2}$, on the other hand, are produced more readily than $\mathrm{HgI}_{2}$, hence their purity is more controlled. In order to ensure the purity of the starting materials for 
synthesis, however, the zone refinement method will be employed to purify commercially obtained $\mathrm{KI}$ and $\mathrm{HgCl}_{2}$. Due to the delay in construction and characterization of the zone refinement furnace, the synthesis of $\mathrm{HgI}_{2}$ has been delayed.

\section{Purification of $\mathbf{H g I}_{2}$ by Sublimation and Melting}

Purification by sublimation was conducted in a five-foot long ampoule (as shown in Figure 13). After the first sublimation run, impurity materials were observed left behind in the stock boat, indicating removal of some impurities by sublimation (see Figure 14). The receiver boat still filled with once purified $\mathrm{HgI}_{2}$ material was then inserted as the stock boat, and a new cleaned boat was inserted as the receiver boat. Another sublimation run was performed, followed again by a third sublimation purification carried out identical to the previous runs. By the end of the third run, there was only an insignificant trace of the impurity materials left in the stock boat.

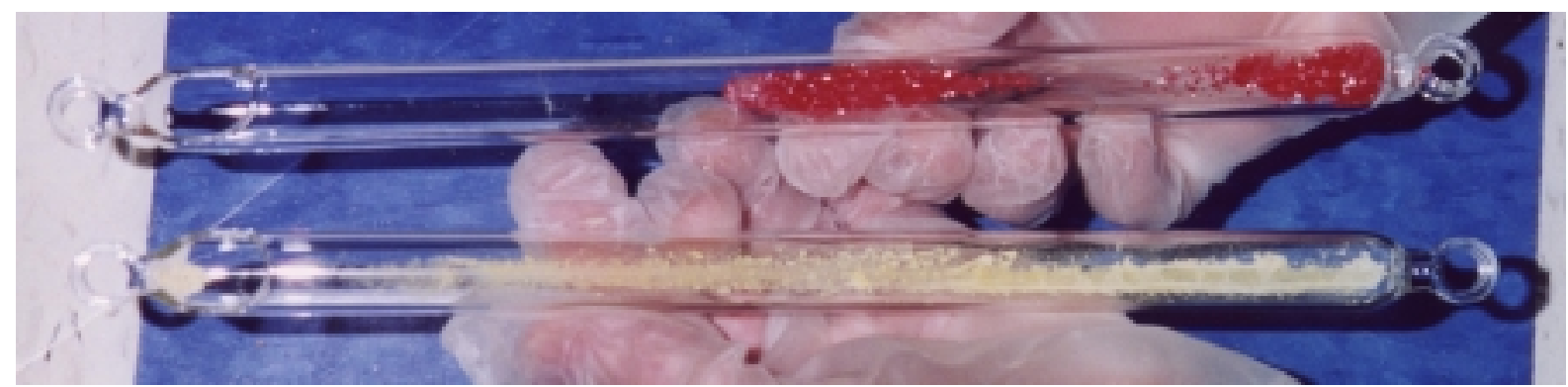

Figure 14: A comparison of the stock (or source) boat (lower) and receiver boat (upper) after a first sublimation purification run. The yellow material left behind in the source boat is a mixture of unwanted impurities. The red material in the receiver boat is $\mathrm{HgI}_{2}$ that has begun the purification process.

Afterwards, the thrice-purified $\mathrm{HgI}_{2}$ material was transferred to another clean ampoule and sealed under vacuum. The material was melted in a high temperature furnace at $300^{\circ} \mathrm{C}$ and allowed to sit for several hours. The process causes heavy metals to plate out of the material and onto the glass wall of the ampoule. Carbonaceous film coating the walls of the melting ampoule indicated that after multiple runs of purification by sublimation carbon was still incorporated in the material. Afterwards, the purified $\mathrm{HgI}_{2}$ was removed from the melt ampoule (see Figure 15) and loaded into growth ampoules for crystal growth. 


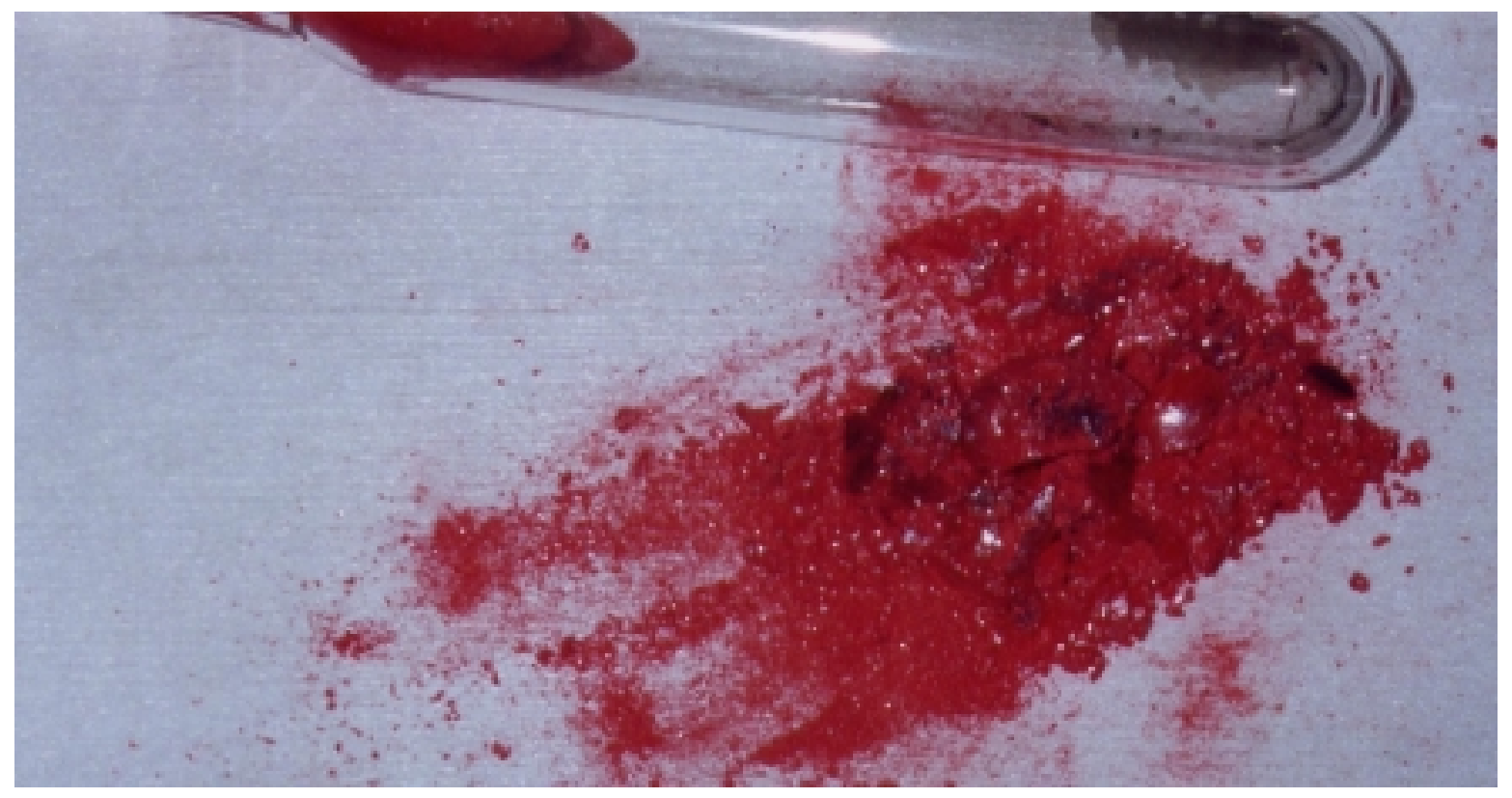

Figure 15: Stock $\mathrm{HgI}_{2}$ material after confined melting. It can be seen that carbonaceous material formed in the material after the run, which could be from the stock material or from contamination during the ampoule sealing procedure. Also, it is observed that impurities are plated onto the ampoule.

\section{Analysis of Purification Results by GDMS}

A sample from each purification run was collected for impurity analysis. Shiva Technologies, Inc. analyzed three samples of $\mathrm{HgI}_{2}$, each originated from different processes (the first was stock material, the second was the result of the first run of purification by sublimation, and the third was the result of purification by melting), by glow discharge mass spectroscopy (GDMS). The results show that most of the trace elements decreased in concentration for each sublimation run. However, some impurities appear to have increased after the melt process, indicating that a source of contamination is the glass ampoule. It becomes apparent that more stringent cleaning processes for the glassware are necessary. It is also speculated that carbon residue from the ampoule sealing process may have contaminated the ampoule. The impurity analysis data can be found in Appendix I.

\section{Growth of $\mathrm{HgI}_{2}$ Crystals in Horizontal Furnaces}

In the experiment using the second new growth design, crystals formed only in the end zone (which was separated from the middle zone by a ring insulator). Several new growth experiments were carried out. The first set of experiments attempted selected growth in a horizontal furnace. This experiment, which also included the use of a new ampoule 
design, employed glass capillary tubes pointing in the direction of the ampoule and blowing dry nitrogen gas unto selected small areas of the ampoule. In the first experiment the flow of nitrogen gas was kept at a constant rate, while in the second one the rate was decreased by a constant increment daily. This incremental temperature changes were implemented to account for an increasing temperature gradient across the growing crystals. In both experiments the source material in the source zone was kept at $120{ }^{\circ} \mathrm{C}$, while the middle zone was kept at $100{ }^{\circ} \mathrm{C}$ and the end zone at $80{ }^{\circ} \mathrm{C}$. As in the previous experiments, the ampoules were evacuated after being filled with source material. Stock material was used as the source material and the growth period for both experiments was five days.

In the first two experiments, crystals formed in and around the small areas cooled down by nitrogen flow through the capillary tubes (as seen in Figures 16 and 17). In ampoule I (Figure 16), which was used in the experiment with a constant nitrogen flow rate, there were smaller polycrystals forming around bigger ones. In ampoule $\mathbf{J}$ (Figure 17), which was used in the experiment with a daily increase of nitrogen flow rate through capillary tubes, polycrystals formed at approximately where the openings of the nitrogen tubes were on the ampoule.

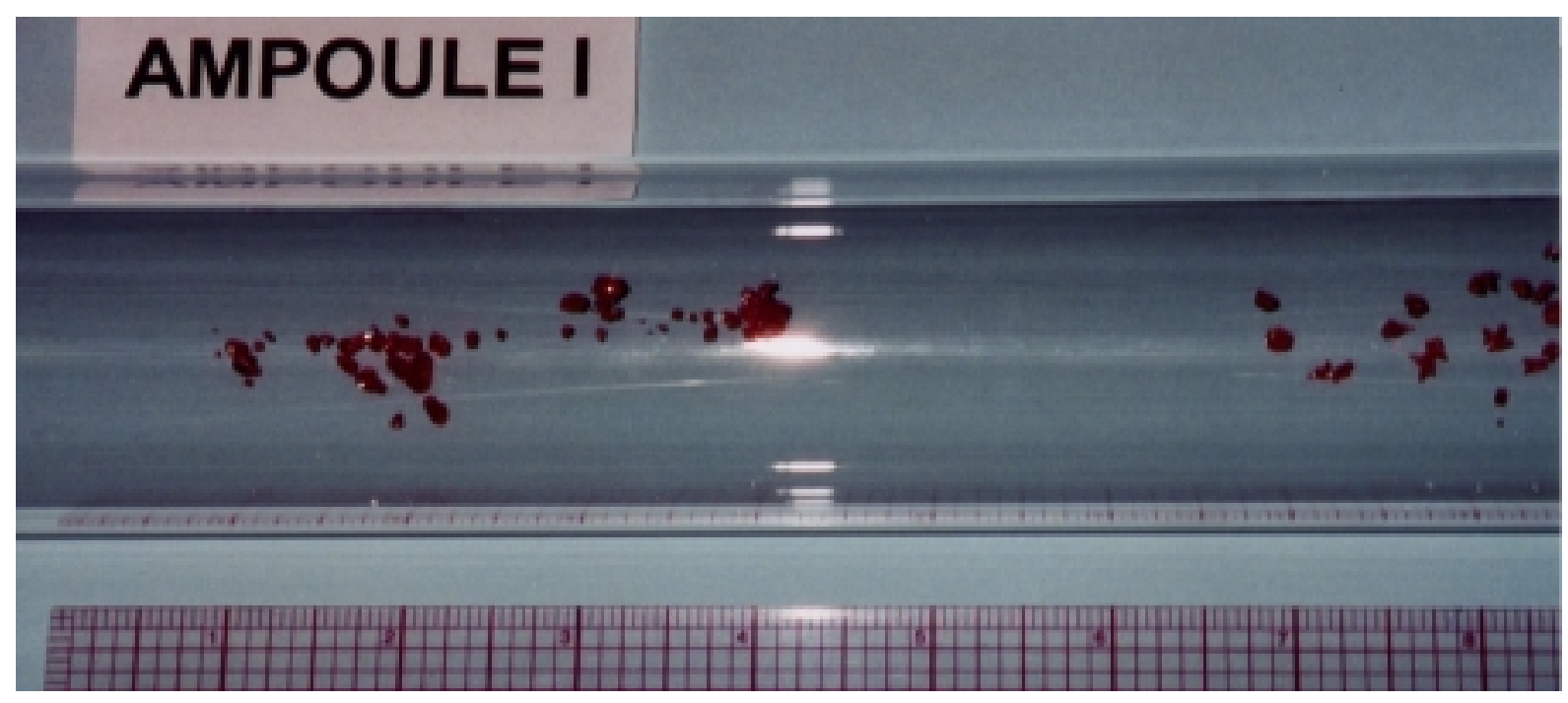

Figure 16: $\mathrm{HgI}_{2}$ crystals growth with nitrogen gas spot cooling. Crystal nucleation produced many single crystals surrounded by polycrystalline material. 


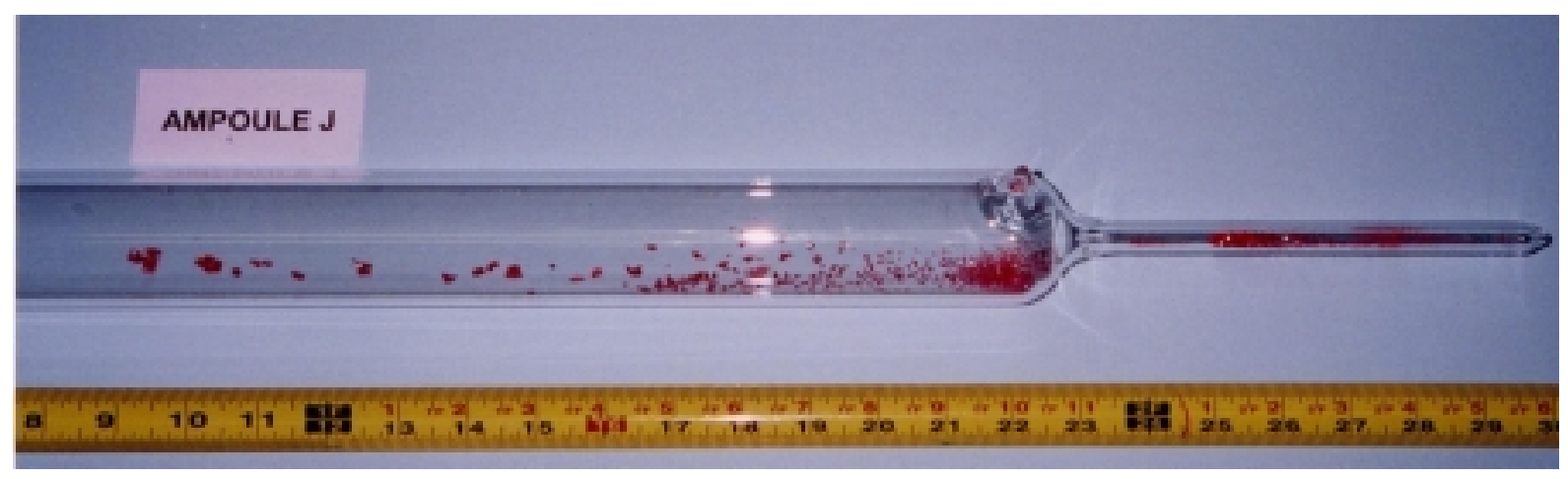

Figure 17: $\mathrm{HgI}_{2}$ crystals growth with nitrogen gas spot cooling.

The second new growth design consisted of the use of two ring zone separators or insulators: one was placed between the source and the middle zones and the second one between the middle and the end zones. These ring insulators also served to keep the ampoule and the furnace coaxial. In this experiment the result of purification by sublimation was used as the source material. It was placed in the source zone kept at $120{ }^{\circ} \mathrm{C}$, while the middle zone was kept at $100{ }^{0} \mathrm{C}$ and the end zone at $90{ }^{\circ} \mathrm{C}$. The growth period for this experiment was five days. The resulting platelets were quite large, exceeding $1.0 \mathrm{~cm}$ in length for many specimens (see Figure 18). Hence, the last method proved to yield the best horizontal growth results thus far. It was noticed that the thermal profile needed to grow crystals from the purified material was consistently different from the unpurified material, requiring a lower temperature profile than previous growth runs. Hence, it is speculated that the impurities in previous starting material raised the vapor pressure of the impure $\mathrm{HgI}_{2}$ material. 


\section{AMPOULE M}

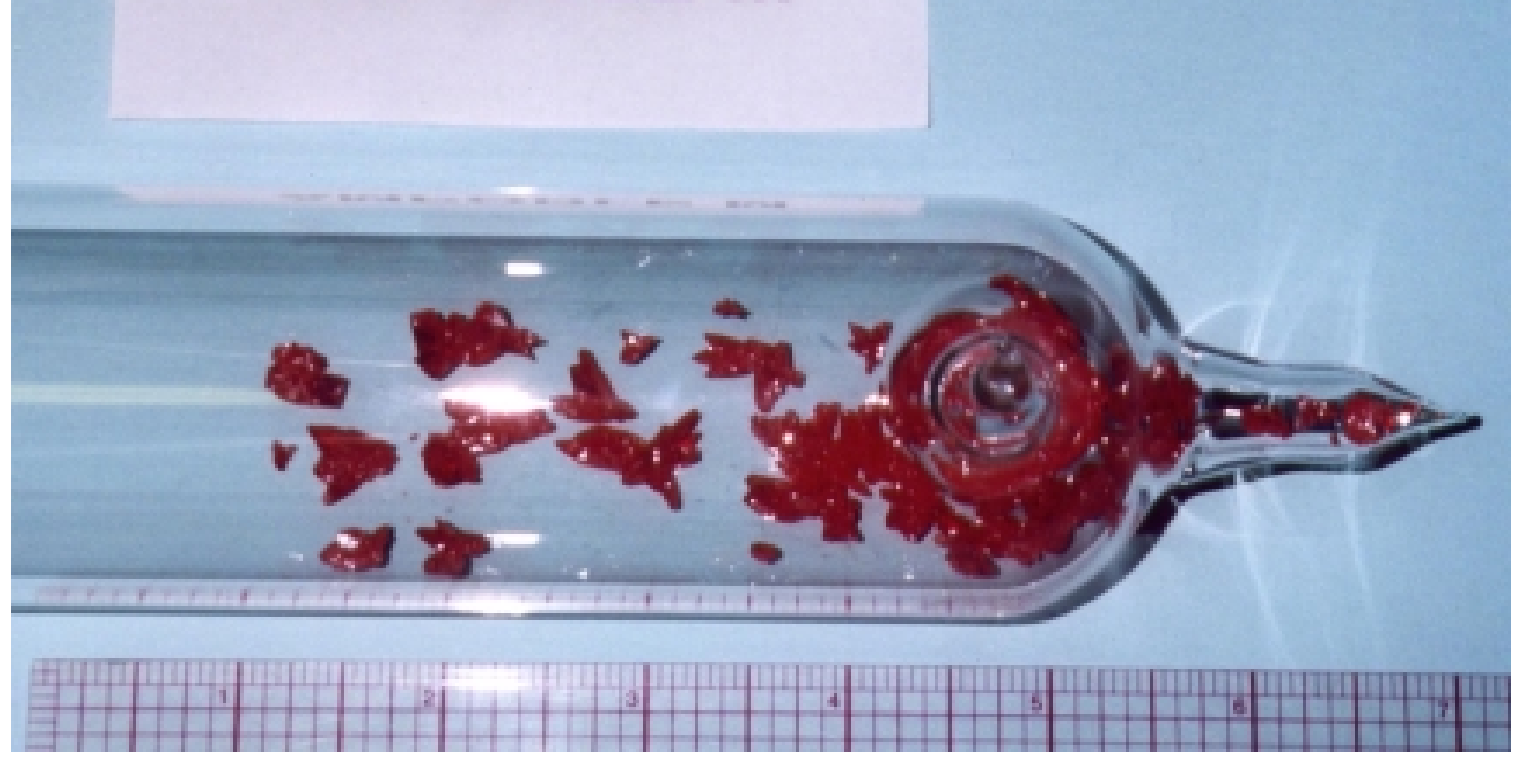

Figure 18: Large $\mathrm{HgI}_{2}$ "platelets" formed from the baffled growth process that used nitrogen capillary tubes and insulating baffles to separate the thermal regions in the furnace. Many of the crystals are 0.5 inches $(1.2 \mathrm{~cm})$ long.

\section{Growth of $\mathrm{HgI}_{2}$ in Vertical Furnaces}

The SMART Laboratory has received several vertical furnaces (which are used to conduct vapor growth of $\mathrm{HgI}_{2}$ crystals by the so-called "EG\&G method") and several corresponding sealed ampoules from Renaissance Instruments and Sandia National Laboratories. Currently four of these furnaces are in working condition and they have been used to grow bulk single crystals in the sealed ampoules.

The vertical $\mathrm{HgI}_{2}$ growth method used is similar to that originally developed by Scholz as the temperature oscillation method [7], and the method allows for the growth of large single crystals. Briefly described, $\mathrm{HgI}_{2}$ that has been cleaned and purified is vacuum sealed into a Pyrex ampoule. There furnace arrangement has three main elements to control for the growth, those being the upper (or axial) heater coil, the lower (or base) heating coil, and a cold finger, generally referred to in the trade as the "sting." 


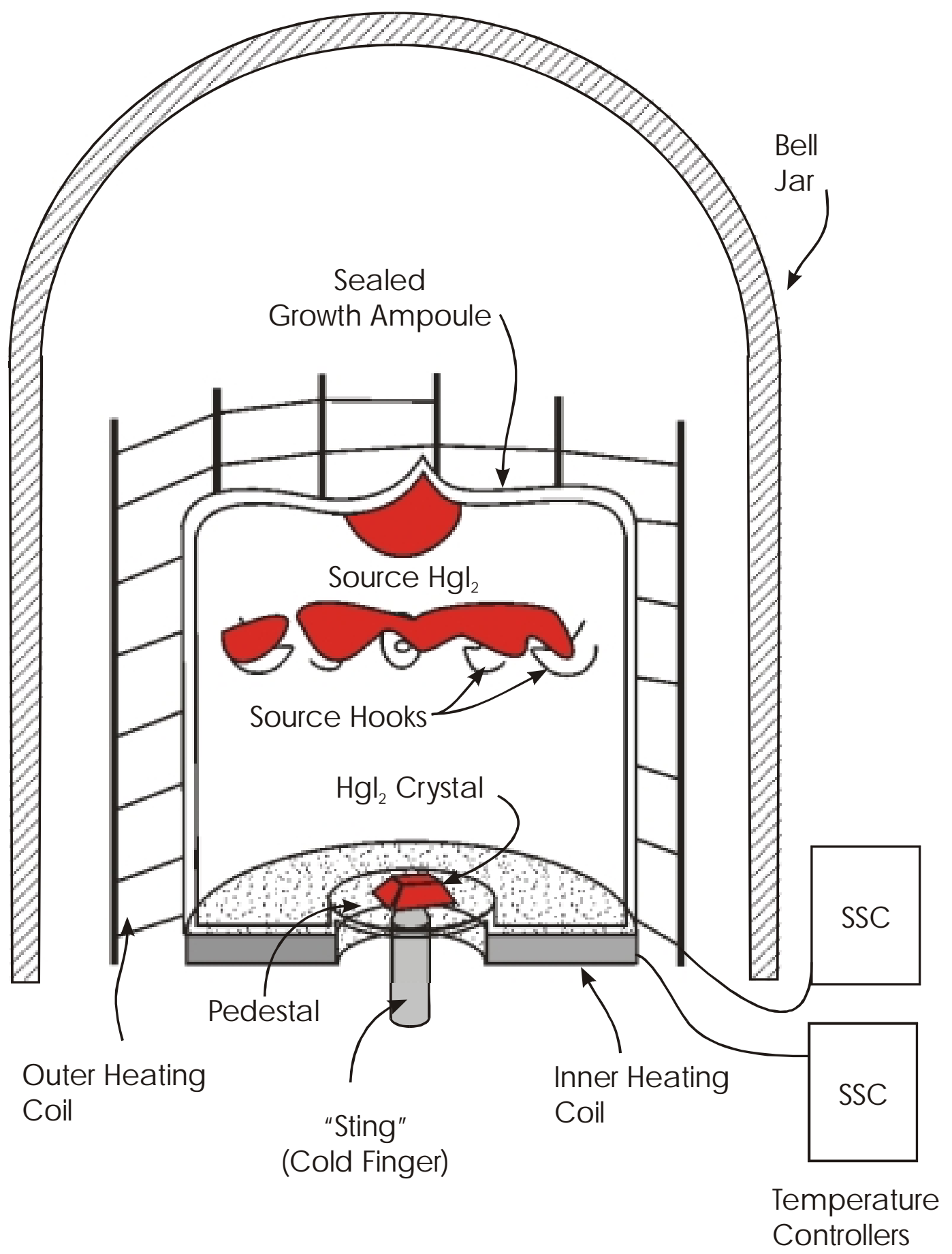

Figure 19: Configuration for vertical growth of $\mathrm{HgI}_{2}$ crystals.

The ampoule is placed in the furnace with the configuration shown in Figure 19, and the inner and outer heating coils are adjusted such that the $\mathrm{HgI}_{2}$ source material accumulates at the top of the ampoule (or on the source hooks). The sting is brought close to a glass pedestal at the bottom part of the ampoule, thus providing the temperature gradient needed to 
nucleate a small single crystal of $\mathrm{HgI}_{2}$ on the pedestal. Once a perfect single crystal has nucleated on the pedestal, the temperature gradient between the source and the crystal adjusted to sustain a constant rate of growth. High quality $\mathrm{HgI}_{2}$ single crystals over $250 \mathrm{~g}$ can be grown with the technique. Figure 20 shows a $\mathrm{HgI}_{2}$ single crystal as formed soon after the nucleation process. As can be seen, the crystal is relatively small. Below the crystal is the cold finger or "sting." Figure 21 shows a much larger $\mathrm{HgI}_{2}$ single crystal several after several weeks of growth, in which the well-formed crystal is quite sizeable and much larger than those produced with the horizontal method.

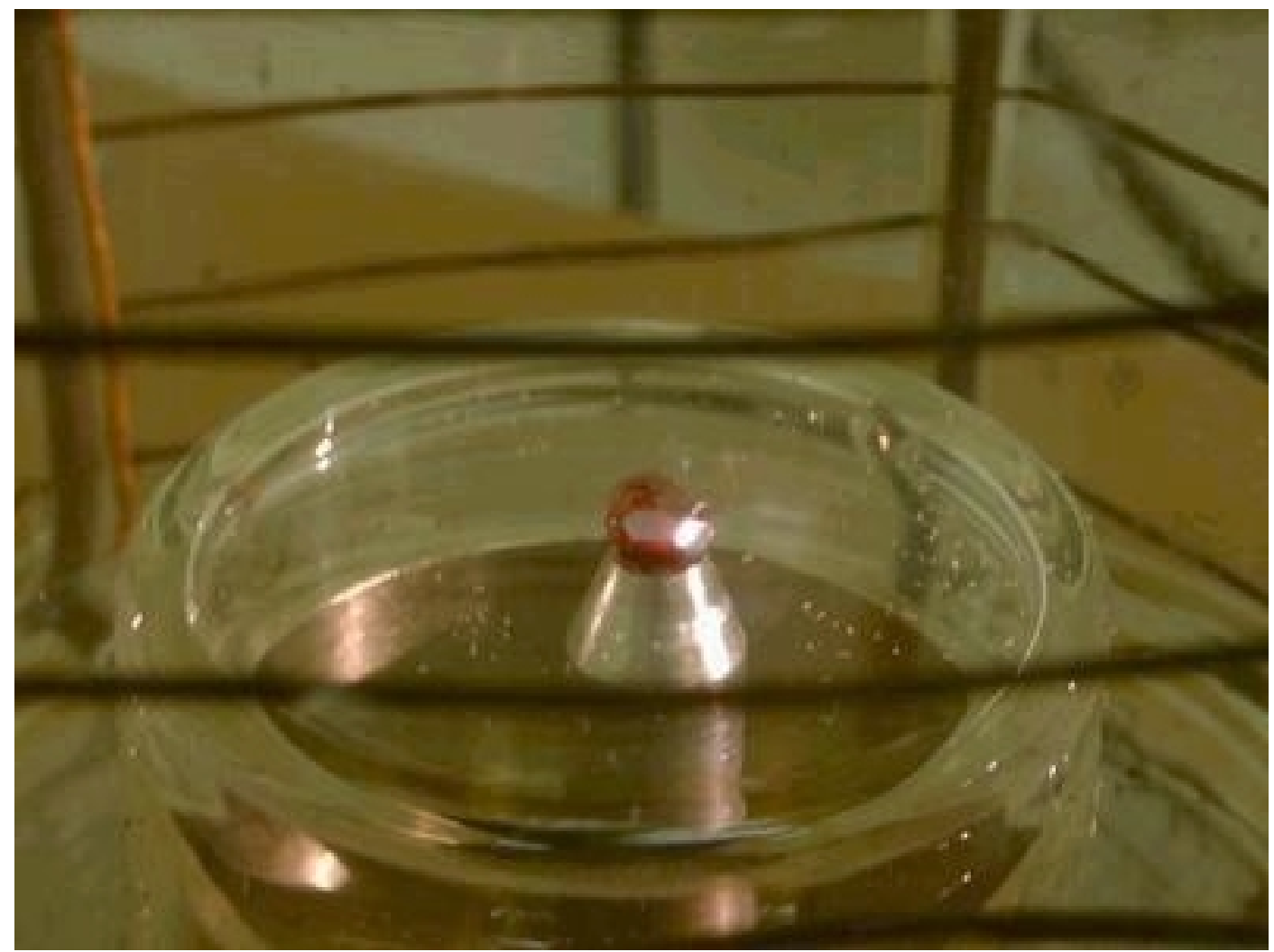

Figure20: A small $\mathrm{HgI}_{2}$ crystal seeded onto a vertical furnace pedestal. The "sting" is the metal cold finger directly below the small $\mathrm{HgI}_{2}$ crystal. 


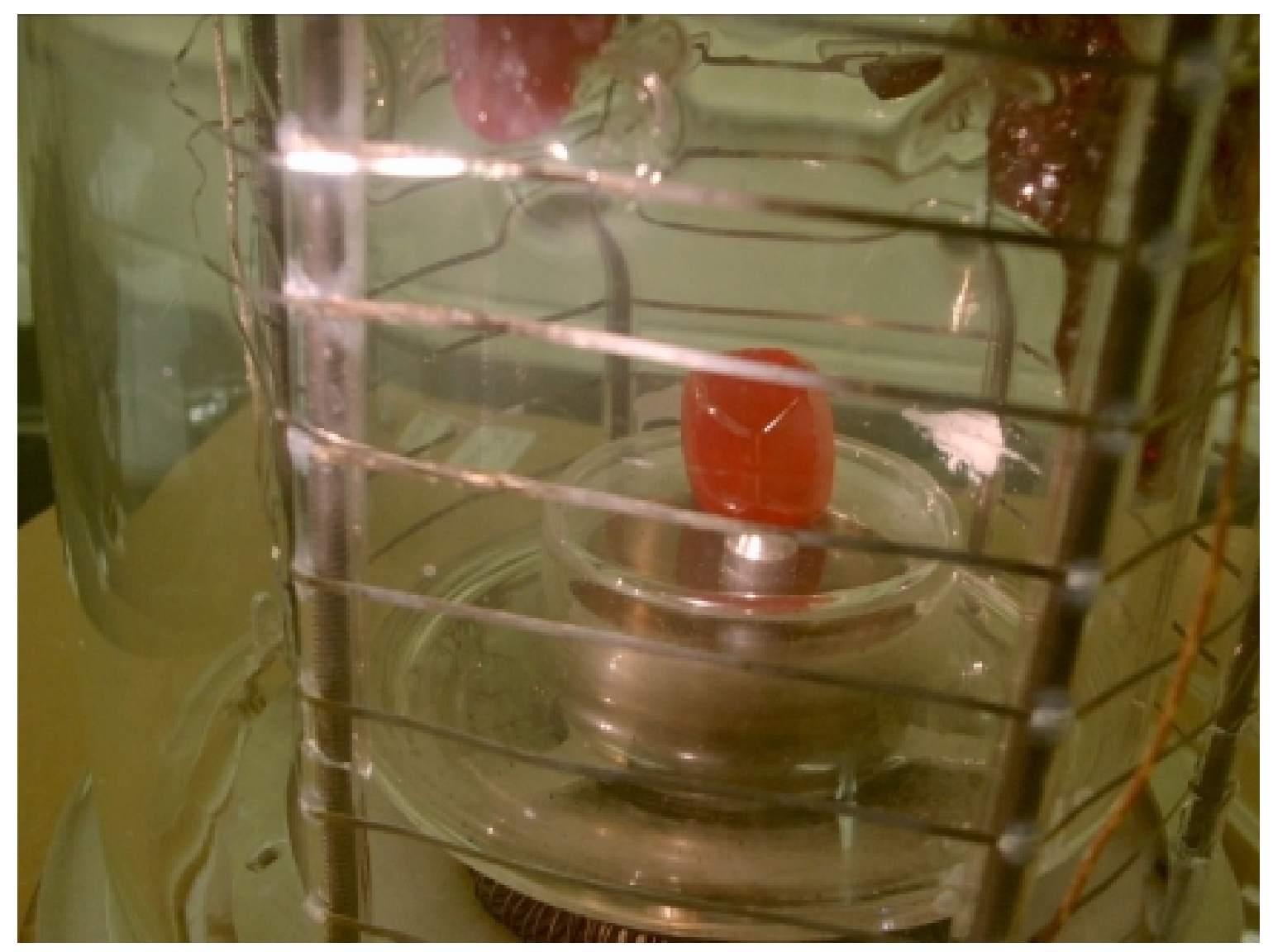

Figure 21: A large $\mathrm{HgI}_{2}$ crystal after several weeks of growth in a vertical furnace.

\section{Future Work}

Horizontal growth ampoules will be loaded with the purified material as previously described. Afterwards, the ampoule will be backfilled (but still under vacuum) with different gases, such as argon, oxygen, hydrogen, nitrogen, and a variety of corrosive gases. The differences in growth will be documented. Crystals large enough for evaluation will be harvested and the material properties evaluated, including resistivity, crystallinity, charge carrier mobility, charge carrier mean free drift times, and ultimately radiation detector performance. Radiation detectors will be fabricated from these crystals showing the most promise and tested with gamma-ray sources for energy resolution and durability.

The growth technique will incorporate several growth passes in the horizontal furnaces acquired from Renaissance Instruments. The growth will proceed as is experimentally determined the best technique. After a few days a growth, however, the thermal profile will be reversed and the growth is continued. This will cause the $\mathrm{HgI}_{2}$ 
material, in vapor form, to pass several times over the optimal crystal growth zone, thereby yielding even larger crystals. The best of the techniques studied will be implemented and modified for the larger furnaces and the resulting crystals studied extensively. The advantages of the horizontal growth technique include (1) scalability to very large diameter furnaces (promising larger crystals) and (2) the realization of multiple crystals per ampoule rather than only one (as typically found with vertical growth techniques).

\section{Bibliography}

1. D.S. McGregor and H. Hermon, Nucl. Instrum. and Meth., A395 (1997) p.101.

2. A. Burger, D. Nason, L. van den Berg, and M. Schieber, Chapter 3 in "Semiconductors and Semimetals," Vol. 43, R.B. James and T.E. Schlesinger, Volume Ed.s; Weber, Willardson and Beer, Series Eds; (Academic Press, San Diego, 1995) p. 86.

3. S. Faile, A. Dabrowski, G. Huth and J. Iwanczyk, J. Cryst. Growth, 50 (1980) p. 752.

4. S. P. Faile, "Vapor Growth of Mercury Iodide for Use as High Energy Detectors," U.S. Patent 4,282,057 (1981).

5. J.D. Phillips, M.W. Lund, J.K. Shurtleff and W.P. Allred, Nucl. Instr. and Meth., A380 (1996) p. 50.

6. Renaissance Instruments, Round Rock, Texas.

7. H. Scholz, Acta Electronica, 17 (1974) p. 69. 


\section{Appendix I: Table of Impurities as Analyzed by GDMS.}

Glow Discharge Mass Spectroscopy Results

\section{Hgl2 \\ $\mathrm{Hg} / 2$}

Stock Material 1st Sublimation Run Melting Run*

\begin{tabular}{|c|c|c|c|}
\hline Element & $\begin{array}{c}\text { Conc. } \\
\text { [ ppm wt. ] }\end{array}$ & $\begin{array}{c}\text { Conc. } \\
\text { [ ppm wt. ] }\end{array}$ & $\begin{array}{c}\text { Conc. } \\
\text { [ ppm wt. ] }\end{array}$ \\
\hline $\mathrm{Li}$ & $<0.01$ & $<0.01$ & $<0.01$ \\
\hline $\mathrm{Be}$ & $<0.005$ & $<0.005$ & $<0.005$ \\
\hline$B$ & 0.02 & 0.18 & 0.04 \\
\hline C & $\sim 12$ & $\sim 2$ & $\sim 15$ \\
\hline $\mathrm{N}$ & $\sim 200$ & $\sim 25$ & $\sim 20$ \\
\hline $\mathrm{O}$ & $\sim 20$ & $\sim 2$ & $\sim 2$ \\
\hline $\mathrm{F}$ & $<0.5$ & $<0.5$ & $<0.5$ \\
\hline $\mathrm{Na}$ & 0.15 & 0.05 & 3 \\
\hline $\mathrm{Mg}$ & 0.04 & $<0.005$ & 0.01 \\
\hline $\mathrm{Al}$ & 0.48 & 0.35 & 0.28 \\
\hline Si & 2 & 1.8 & 0.38 \\
\hline$P$ & 0.02 & $<0.005$ & $<0.005$ \\
\hline$S$ & 8 & 0.35 & 0.35 \\
\hline $\mathrm{Cl}$ & 35 & 14 & 2 \\
\hline $\mathrm{K}$ & 20 & $<0.05$ & 0.15 \\
\hline $\mathrm{Ca}$ & 0.5 & $<0.05$ & $<0.05$ \\
\hline $\mathrm{Sc}$ & $<0.001$ & $<0.001$ & $<0.001$ \\
\hline $\mathrm{Ti}$ & 0.75 & 0.17 & 0.03 \\
\hline $\mathrm{V}$ & $<0.005$ & $<0.005$ & $<0.005$ \\
\hline $\mathrm{Cr}$ & 0.02 & 0.02 & $<0.01$ \\
\hline $\mathrm{Mn}$ & $<0.005$ & $<0.005$ & $<0.005$ \\
\hline $\mathrm{Fe}$ & 1.7 & 0.28 & 0.28 \\
\hline Co & $<0.005$ & $<0.005$ & $<0.005$ \\
\hline $\mathrm{Ni}$ & 0.06 & $<0.01$ & $<0.01$ \\
\hline $\mathrm{Cu}$ & Sec. Cathode & Sec. Cathode & Sec. Cathode \\
\hline $\mathrm{Zn}$ & $<0.5$ & $<0.5$ & $<0.5$ \\
\hline $\mathrm{Ga}$ & $<0.05$ & $<0.05$ & $<0.05$ \\
\hline $\mathrm{Ge}$ & $<0.05$ & $<0.05$ & $<0.05$ \\
\hline As & 0.18 & 0.22 & 0.08 \\
\hline $\mathrm{Se}$ & $<0.5$ & $<0.5$ & $<0.5$ \\
\hline $\mathrm{Br}$ & 1.8 & 1.2 & 0.45 \\
\hline $\mathrm{Rb}$ & $<0.005$ & $<0.005$ & $<0.005$ \\
\hline $\mathrm{Sr}$ & $<0.005$ & $<0.005$ & $<0.005$ \\
\hline$Y$ & $<0.005$ & $<0.005$ & $<0.005$ \\
\hline $\mathrm{Zr}$ & $<0.005$ & $<0.005$ & $<0.005$ \\
\hline $\mathrm{Nb}$ & $<0.05$ & $<0.05$ & $<0.05$ \\
\hline Mo & $<0.05$ & $<0.05$ & $<0.05$ \\
\hline
\end{tabular}




\begin{tabular}{|c|c|c|c|}
\hline $\mathrm{Ru}$ & $<0.05$ & $<0.05$ & $<0.05$ \\
\hline $\mathrm{Rh}$ & $<0.5$ & $<0.5$ & $<0.5$ \\
\hline $\mathrm{Pd}$ & $<0.05$ & $<0.05$ & $<0.05$ \\
\hline $\mathrm{Ag}$ & $=<30$ & $=<2$ & $=<0.7$ \\
\hline $\mathrm{Cd}$ & $<0.5$ & $<0.5$ & $<0.5$ \\
\hline $\ln$ & 0.15 & $<0.005$ & $<0.005$ \\
\hline Sn & 0.2 & $<0.01$ & $<0.01$ \\
\hline $\mathrm{Sb}$ & 0.75 & $<0.01$ & $<0.01$ \\
\hline $\mathrm{Te}$ & $<0.5$ & $<0.5$ & $<0.5$ \\
\hline I & Matrix & Matrix & Matrix \\
\hline Cs & $<0.005$ & $<0.005$ & $<0.005$ \\
\hline $\mathrm{Ba}$ & $<0.005$ & $<0.005$ & $<0.005$ \\
\hline $\mathrm{La}$ & $<0.005$ & $<0.005$ & $<0.005$ \\
\hline $\mathrm{Ce}$ & $<0.005$ & $<0.005$ & $<0.005$ \\
\hline $\operatorname{Pr}$ & $<0.005$ & $<0.005$ & $<0.005$ \\
\hline $\mathrm{Nd}$ & $<0.005$ & $<0.005$ & $<0.005$ \\
\hline $\mathrm{Sm}$ & $<0.005$ & $<0.005$ & $<0.005$ \\
\hline $\mathrm{Eu}$ & $<0.005$ & $<0.005$ & $<0.005$ \\
\hline $\mathrm{Gd}$ & $<0.005$ & $<0.005$ & $<0.005$ \\
\hline $\mathrm{Tb}$ & $<0.005$ & $<0.005$ & $<0.005$ \\
\hline Dy & $<0.005$ & $<0.005$ & $<0.005$ \\
\hline Ho & $<0.005$ & $<0.005$ & $<0.005$ \\
\hline $\mathrm{Er}$ & $<0.005$ & $<0.005$ & $<0.005$ \\
\hline $\mathrm{Tm}$ & $<0.005$ & $<0.005$ & $<0.005$ \\
\hline $\mathrm{Yb}$ & $<0.005$ & $<0.005$ & $<0.005$ \\
\hline $\mathrm{Lu}$ & $<0.005$ & $<0.005$ & $<0.005$ \\
\hline $\mathrm{Hf}$ & $<0.005$ & $<0.005$ & $<0.005$ \\
\hline $\mathrm{Ta}$ & Source & Source & Source \\
\hline W & 0.04 & $<0.01$ & $<0.01$ \\
\hline $\mathrm{Re}$ & $<0.01$ & $<0.01$ & $<0.01$ \\
\hline Os & $<0.01$ & $<0.01$ & $<0.01$ \\
\hline $\mathrm{Ir}$ & $<0.01$ & $<0.01$ & $<0.01$ \\
\hline $\mathrm{Pt}$ & $<0.05$ & $<0.05$ & $<0.05$ \\
\hline $\mathrm{Au}$ & $<0.5$ & $<0.5$ & $<0.5$ \\
\hline $\mathrm{Hg}$ & Matrix & Matrix & Matrix \\
\hline $\mathrm{TI}$ & $<0.05$ & $<0.05$ & $<0.05$ \\
\hline $\mathrm{Pb}$ & 0.12 & $<0.05$ & $<0.05$ \\
\hline $\mathrm{Bi}$ & $<0.05$ & $<0.05$ & $<0.05$ \\
\hline Th & $<0.01$ & $<0.01$ & $<0.01$ \\
\hline$U$ & $<0.01$ & $<0.01$ & $<0.01$ \\
\hline
\end{tabular}

* (HgI2 sample was tainted with carbon compounded on the glass surface during melting). 